\title{
Lower Bound on Testing Membership to a Polyhedron by Algebraic Decision and Computation Trees*
}

\author{
D. Grigoriev, ${ }^{1}$ M. Karpinski, ${ }^{2}$ and N. Vorobjov ${ }^{3}$ \\ 'Departments of Computer Science and Mathematics, Penn State University, \\ University Park, PA 16802, USA \\ dima@cse.psu.edu \\ ${ }^{2}$ Department of Computer Science, University of Bonn, \\ 53117 Bonn, Germany, \\ and \\ International Computer Science Institute, \\ Berkeley, CA 94704, USA \\ marek@cs.bonn.edu \\ ${ }^{3}$ School of Mathematical Sciences, University of Bath, \\ Bath, Avon BA2 7AY, England \\ nnv@maths.bath.ac.uk
}

\begin{abstract}
We introduce a new method of proving lower bounds on the depth of algebraic $d$-degree decision (resp. computation) trees and apply it to prove a lower bound $\Omega(\log N)$ (resp. $\Omega(\log N / \log \log N)$ ) for testing membership to an $n$-dimensional convex polyhedron having $N$ faces of all dimensions, provided that $N>(n d)^{\Omega(n)}$ (resp. $N>n^{\Omega(n)}$ ). This bound apparently does not follow from the methods developed by Ben-Or, Björner, Lovasz, and Yao [1], [4], [24] because topological invariants used in these methods become trivial for convex polyhedra.
\end{abstract}

\section{Introduction}

A problem of testing membership to a semialgebraic set $\Sigma$ was considered by many authors (see, e.g., [1]-[5], [9], [17], [19], [23]-[25], and the references there). We consider a problem of testing membership to a convex polyhedron $P$ in $n$-dimensional space $\mathbf{R}^{n}$.

* The research of D. Grigoriev was supported in part by NSF Grant CCR-9424358. M. Karpinski was supported in part by DFG Grant KA673/4-1, by the ESPRIT BR Grants 7097 and EC-US 030, and by the Volkswagen-Stiftung. 
Let $P$ have $N$ faces of all the dimensions. In [17] it was shown, in particular, that for this problem an $O(\log N) n^{O(1)}$ upper bound is valid for the depth of linear decision trees. In [25] a lower bound $\Omega(\log N)$ was obtained. A similar question was open for algebraic decision and computation trees. In [9] we proved a lower bound $\Omega(\log N)$ for the depth of algebraic decision trees testing membership to $P$, provided that $N>(d n)^{\Omega\left(n^{2}\right)}$. In the present paper we weaken the latter assumption to $N>(d n)^{\Omega(n)}$ and also prove a lower bound $\Omega(\log N / \log \log N)$ for algebraic computation trees, provided that $N>n^{\Omega(n)}$. In this new form the bound looks plausible enough to be applicable to polyhedra given by $2^{O(n)}$ linear constraints (like in the "knapsack" problem), thus having $2^{O\left(n^{2}\right)}$ faces. In this paper we apply the obtained lower bound to a concrete class of polyhedra given by $\Omega\left(n^{2}\right)$ linear constraints and with $n^{\Omega(n)}$ faces.

In [12] the lower bound $\Omega(\sqrt{\log N})$ was proved for the Pfaffian computation tree model. At gates this model uses Pfaffian functions, the latter include all major elementary transcendental and algebraic functions.

Several topological methods were introduced for obtaining lower bounds for the complexity of testing membership to $\Sigma$ by linear decision trees, algebraic decision trees, and algebraic computation trees (the definitions can be found in, e.g., [1]).

In [1] a lower bound $\Omega(\log C)$ was proved for the most powerful among the computational models considered in this area, namely algebraic computation trees, where $C$ is the number of connected components of $\Sigma$ or of the complement of $\Sigma$. After that, in [4], a lower bound $\Omega(\log \chi)$ for linear decision trees was proved, where $\chi$ is the Euler characteristic of $\Sigma$, in [23] this lower bound was extended to algebraic decision and computation trees. A stronger lower bound $\Omega(\log B)$ was proved later in [3] and [2] for linear decision trees, where $B$ is the sum of the Betti numbers of $\Sigma$ (obviously, $C, \chi \leq B$ ). In [24] the latter lower bound was extended to the algebraic decision and computation trees. In [19] the lower bound $\Omega(\sqrt{\log (B) / n})$ was proved for the parallel complexity model.

Unfortunately, all the topological tools mentioned fail when $\Sigma$ is a convex polyhedron, because $B=1$ in this situation. The same is true for the method developed in [4] for linear decision trees, based on the minimal number of convex polyhedra onto which $\Sigma$ can be partitioned.

To handle the case of a convex polyhedron, in Sections 1 and 3 we introduce another approach which differs drastically from [9]. Let $W$ be a semialgebraic set accepted by a branch of an algebraic decision tree. In Section 3 we make an "infinitesimal perturbation" of $W$ which transforms this set into a smooth hypersurface. Then we describe the semialgebraic subset of all the points of the hypersurface in which all its principal curvatures are "infinitely large" (the set $\mathcal{K}_{0}$ in Section 3 ). We also construct a more general set $\mathcal{K}_{i}$ (for each $0 \leq i \leq n-1$ ) of the points with infinitely large curvatures in the intersections with the shifts of a fixed $(n-i)$-dimensional plane. Section 1 provides a short system of inequalities for determining $\mathcal{K}_{i}$. It is done by developing an explicit symbolic calculus for principal curvatures.

In Section 2 we introduce some necessary notions concerning infinitesimals and apply them to define the "standard part" $K_{i}=\operatorname{st}\left(\mathcal{K}_{i}\right) \subset \mathbf{R}^{n}$. We show (the corollary to Lemma 5 in Section 3) that to obtain the required bound for the number of $i$-faces $P_{i}$ of $P$ such that $\operatorname{dim}\left(P_{i} \cap W\right)=i$ it is sufficient to estimate the number of faces $P_{i}$ with $\operatorname{dim}\left(P_{i} \cap K_{i}\right)=i$. In Section 4 we reduce the latter bound to an estimate of the number of 
local maxima of a generic linear function $L$ on $\mathcal{K}_{i}$ with the help of a Whitney stratification of $K_{i}$. To estimate these local maxima in Section 5 we introduce another infinitesimal perturbation of $\mathcal{K}_{i}$ and obtain a new smooth hypersurface. At this point a difficulty arises due to the fact that $\mathcal{K}_{i}$ (and, therefore, the related smooth hypersurface) are defined by systems of inequalities involving algebraic functions, rather than polynomials, because in the expressions for curvatures (in Section 1) square roots of polynomials appear. We represent the set of local maxima of $L$ on the smooth hypersurface by a formula of the first-order theory of real closed fields with merely existential quantifiers and a quantifierfree part $\Phi$. In Section 5 we estimate (invoking Milnor's bound [18] in the usual way) the number of the connected components of the semialgebraic set defined by $\Phi$.

In Section 6 we describe a particular class of polyhedra (dual to cyclic polyhedra [16]) having large numbers of faces, for which Theorem 1 provides a nontrivial lower bound.

In Section 7 we give an outline of the proof of the complexity lower bound for testing membership to a polyhedron by computation (rather than decision) trees; see Theorem $1^{\prime}$ below. A complete proof is given only for the case of decision trees because for computation trees it is similar with the addition of some extra technical details.

Now we formulate precisely the main result. We consider algebraic decision trees of a fixed degree $d$ (see, e.g., [1] and [24]). Suppose that such a tree $T$, of depth $k$, tests a membership to a convex polyhedron $P \subset \mathbf{R}^{n}$. Denote by $N$ the number of faces of $P$ of all dimensions from zero to $n-1$. In this paper we agree that a face is "open," i.e., does not contain faces of smaller dimensions.

\section{Theorem 1.}

$$
k \geq \Omega(\log N)
$$

provided that $N \geq(d n)^{c n}$ for a suitable $c>0$.

We fix a branch of $T$ which returns "yes." Denote by $f_{i} \in \mathbf{R}\left[X_{1}, \ldots, X_{n}\right], 1 \leq$ $i \leq k$, the polynomials of degree $\operatorname{deg}\left(f_{i}\right) \leq d$, attached to the vertices of $T$ along the fixed branch. Without loss of generality, we can assume that the corresponding signs of polynomials along the branch are

$$
f_{1}=\cdots=f_{k_{1}}=0, \quad f_{k_{1}+1}>0, \ldots, f_{k}>0 .
$$

Then the (accepted) semialgebraic set

$$
W=\left\{f_{1}=\cdots=f_{k_{1}}=0, f_{k_{1}+1}>0, \ldots, f_{k}>0\right\}
$$

lies in $P$.

Our main technical tool is the following theorem.

Theorem 2. The number offaces $P^{\prime}$ of $P$ such that $\operatorname{dim}\left(P^{\prime}\right)=\operatorname{dim}\left(P^{\prime} \cap W\right)$ is bounded from above by $(k n d)^{O(n)}$.

We deduce Theorem 1 from Theorem 2. 
For each face $P^{\prime}$ of $P$ there exists at least one branch of the tree $T$ with the output "yes" and having an accepted set $W_{1} \subset \mathbf{R}^{n}$ such that

$$
\operatorname{dim}\left(W_{1} \cap P^{\prime}\right)=\operatorname{dim}\left(P^{\prime}\right)
$$

Since there are at most $3^{k}$ different branches of $T$, the inequality

$$
N<3^{k}(k n d)^{O(n)}
$$

follows from Theorem 2. This inequality and the assumption $N>(d n)^{c n}$ (for a suitable c) imply $k \geq \Omega(\log N)$, which proves Theorem 1 .

Note that in the case $k_{1}=0$ for an open set $W$ and each face $P^{\prime}$ of $P$ we have $P^{\prime} \cap W=\emptyset$. Thus in what follows we can suppose that $k_{1} \geq 1$.

Finally we formulate a complexity lower bound for algebraic computation trees. Let $k$ now be the depth of a computation tree, which tests membership to $P$.

Theorem 1'.

$$
k \geq \Omega\left(\frac{\log N}{\log \log N}\right)
$$

provided that $N \geq n^{c_{1} n}$ for a suitable $c_{1}>0$.

\section{Computer Algebra for Curvatures}

Let a polynomial $F \in \mathbf{R}\left[X_{1}, \ldots, X_{n}\right]$ with $\operatorname{deg}(F)<d$. Assume that at a point $x \in\{F=$ $0\} \subset \mathbf{R}^{n}$ the gradient $\operatorname{grad}_{x}(F)=\left(\partial F / \partial X_{1}, \ldots, \partial F / \partial X_{n}\right)(x) \neq 0$. Then, according to the implicit function theorem, the real algebraic variety $\{F=0\} \subset \mathbf{R}^{n}$ is a smooth hypersurface in a neighborhood of $x$.

Fix a point $x \in\{F=0\}$. Consider a linear transformation $X \rightarrow A_{x} X+x$, where $A_{x}$ is an arbitrary orthogonal matrix such that

$$
u_{1}=A_{x} e_{1}+x=\frac{\operatorname{grad}_{x}(F)}{\left\|\operatorname{grad}_{x}(F)\right\|}
$$

is the normalized gradient and $e_{1}, \ldots, e_{n}$ is the coordinate basis at the origin. Then the linear hull of vectors $u_{j}=A_{x} e_{j}+x, 2 \leq j \leq n$, is the tangent space $T_{x}$ to $\{F=0\}$ at $x$.

Denote by $U_{1}, \ldots, U_{n}$ the coordinate variables in the basis $u_{1}, \ldots, u_{n}$. By the implicit function theorem, there exists a smooth function $H_{x}\left(U_{2}, \ldots, U_{n}\right)$ defined in a neighborhood of $x$ on $T_{x}$ such that $\{F=0\}=\left\{U_{1}=H_{x}\left(U_{2}, \ldots, U_{n}\right)\right\}$ in this neighborhood.

Let $\operatorname{grad}_{x}(F)=\left(\tilde{\alpha}_{1}, \ldots, \tilde{\alpha}_{n}\right)$ with $\tilde{\alpha}_{i_{0}} \neq 0$. Take any permutation $\pi_{i_{0}}$ of $\{1, \ldots, n\}$ such that $\pi_{i_{0}}(1)=i_{0}$. Denote $\left(\alpha_{1}, \ldots, \alpha_{n}\right)=\left(\tilde{\alpha}_{\pi_{i_{0}}(1)}, \ldots, \tilde{\alpha}_{\pi_{i_{0}}(n)}\right)$ (thus $\left.\alpha_{1} \neq 0\right)$ and $\beta_{i}=\sqrt{\alpha_{1}^{2}+\cdots+\alpha_{i}^{2}}, 1 \leq i \leq n$. Obviously $\beta_{i}>0$ and $\beta_{n}=\left\|\operatorname{grad}_{x}(F)\right\|$. 
As $A_{x}$ the following product of $(n-1)$ orthogonal matrices can be taken:

$$
\prod_{0 \leq k \leq n-2}\left(\begin{array}{cccccccc}
\beta_{n-k-1} / \beta_{n-k} & 0 & \cdots & 0 & \alpha_{n-k} / \beta_{n-k} & 0 & \cdots & 0 \\
0 & 1 & \cdots & 0 & 0 & 0 & \cdots & 0 \\
\vdots & \vdots & \ddots & \vdots & \vdots & \vdots & \ddots & \vdots \\
0 & 0 & \cdots & 1 & 0 & 0 & \cdots & 0 \\
-\alpha_{n-k} / \beta_{n-k} & 0 & \cdots & 0 & \beta_{n-k-1} / \beta_{n-k} & 0 & \cdots & 0 \\
0 & 0 & \cdots & 0 & 0 & 1 & \cdots & 0 \\
\vdots & \vdots & \ddots & \vdots & \vdots & \vdots & \ddots & \vdots \\
0 & 0 & \cdots & 0 & 0 & 0 & \cdots & 1
\end{array}\right)
$$

(in the $k$ th matrix of this product the element $\beta_{n-k-1} / \beta_{n-k}$ occurs at positions $(1,1)$ and $(n-k, n-k))$.

Denote $F_{x}\left(U_{1}, \ldots, U_{n}\right)=F\left(A_{x}^{T}\left(U_{1}, \ldots, U_{n}\right)+x\right)$. Differentiating this function twice and taking into the account that $F_{x}\left(H_{x}\left(U_{2}, \ldots, U_{n}\right), U_{2}, \ldots, U_{n}\right)=0$ in a neighborhood of $x$ in $T_{x}$ we get

$$
\frac{\partial^{2} F_{x}}{\partial U_{1} \partial U_{j}} \frac{\partial H_{x}}{\partial U_{i}}+\frac{\partial F_{x}}{\partial U_{1}} \frac{\partial^{2} H_{x}}{\partial U_{i} \partial U_{j}}+\frac{\partial^{2} F_{x}}{\partial U_{i} \partial U_{j}}=0
$$

for $2 \leq i, j \leq n$.

Since

$$
\left.\frac{\partial H_{x}}{\partial U_{i}}\right|_{\left(U_{2}, \ldots, U_{n}\right)=0}=0 \text { and }\left.\frac{\partial F_{x}}{\partial U_{1}}\right|_{\left(U_{1}, \ldots, U_{n}\right)=0}=\left\|\operatorname{grad}_{x}(F)\right\| \neq 0,
$$

evaluating equality (1) at $x$ (i.e., substituting $\left(U_{1}, \ldots, U_{n}\right)=0$ ) we obtain (see [18])

$$
\left.\left(\frac{\partial^{2} H_{x}}{\partial U_{i} \partial U_{j}}\right)\right|_{\left(U_{2}, \ldots, U_{n}\right)=0}=\left.\left(\left\|\operatorname{grad}_{x}(F)\right\|\right)^{-1}\left(\frac{\partial^{2} F_{x}}{\partial U_{i} \partial U_{j}}\right)\right|_{\left(U_{1}, \ldots, U_{n}\right)=0} .
$$

Introduce the symmetric $(n-1) \times(n-1)$ matrix (the matrix of the Weingarten map [22, Chapter 9])

$$
\mathcal{H}_{x}=\left.\left(\frac{\partial^{2} H_{x}}{\partial U_{i} \partial U_{j}}\right)\right|_{\left(U_{2}, \ldots, U_{n}\right)=0}
$$

Its eigenvalues $\lambda_{2}, \ldots, \lambda_{n}$ belong to $\mathbf{R}$ and are called the principal curvatures of the hypersurface $\{F=0\}$ at $x$ [22, Chapter 12]).

Now we describe symbolically the set of all points $x$ with all principal curvatures greater than some parameter $\kappa$.

Denote by $\chi(Z)$ the characteristic polynomial of the matrix $\mathcal{H}_{x}$. The roots of $\chi$ are exactly $\lambda_{2}, \ldots, \lambda_{n}$. Due to Sturm theorem, every $\lambda_{2}, \ldots, \lambda_{n}$ is greater than $\kappa$ if and only if $\chi_{l}(\kappa) \chi_{l+1}(\kappa)<0,0 \leq l \leq n-2$, where $\chi_{0}=\chi, \chi_{1}=\chi_{0}^{\prime}$, and $\chi_{2}, \ldots, \chi_{n-1}$ is the polynomial remainder sequence of $\chi_{0}, \chi_{1}$ [15]. Obviously $\operatorname{deg}_{z}\left(\chi_{l}\right)=n-l-1$.

Observe that every element of the matrix $A_{x}$ can be represented as a fraction $\gamma_{1} / \gamma_{2}$ where $\gamma_{2}=\beta_{1}^{\nu_{1}} \cdots \beta_{n}^{\nu_{n}}, v_{1} \geq 0, \ldots, v_{n} \geq 0$, are integers and

$$
\gamma_{1}=\Gamma\left(\beta_{1}, \ldots, \beta_{n-1}, X_{1}, \ldots, X_{n}\right)
$$


is a polynomial in

$$
\beta_{1}\left(X_{1}, \ldots, X_{n}\right), \ldots, \beta_{n-1}\left(X_{1}, \ldots, X_{n}\right), X_{1}, \ldots, X_{n}
$$

with $\Gamma \in \mathbf{R}\left[Z_{1}, \ldots, Z_{n-1}, X_{1}, \ldots, X_{n}\right]$. Moreover, $v_{1}+\cdots+v_{n} \leq 2(n-1)$ and $\operatorname{deg}(\Gamma) \leq d(n-1)$. Hence all elements of $A_{x}$ are algebraic functions in $X_{1}, \ldots, X_{n}$ of quadratic-irrational type. By the degree of such a quadratic-irrational function we mean

$$
\max \left\{\operatorname{deg}(\Gamma), v_{1}+\cdots+v_{n}\right\}
$$

Since an inequality for a fraction could be rewritten as a system of inequalities for its numerator and denominator, in what follows we deal with more special algebraic functions in $X_{1}, \ldots, X_{n}$, namely of the type $\gamma_{1}$.

Formula (2) and Habicht's theorem [15] imply that $\operatorname{deg}\left(\chi_{l}\right) \leq(n d)^{O(1)}$.

We summarize a description of the set of all points with large principal curvatures in the following lemma.

Lemma 1. Fix $1 \leq i_{0} \leq n$. The set of all points $x \in\{F=0\}$ such that $\operatorname{grad}_{x}(F)=$ $\left(\hat{\alpha}_{1}, \ldots, \hat{\alpha}_{n}\right)$ has $\hat{\alpha}_{i_{0}} \neq 0$ and all principal curvatures of the hypersuface $\{F=0\}$ at $x$ are greater than $\kappa$, can be represented as $\left\{F=0, g_{1}>0, \ldots, g_{n}>0\right\}$. Here $g_{1}=\hat{\alpha}_{i_{0}}^{2}, g_{2}, \ldots, g_{n}$ are polynomials in $\kappa$ of degree at most $2 n$ with coefficients being quadratic-irrational algebraic functions (see above) of degree less than (nd) ${ }^{O(1)}$.

Remark. Observe that a set given by a system of inequalities involving real algebraic functions is semialgebraic. Hence the set introduced in Lemma 1 is semialgebraic.

\section{Calculus with Infinitesimals}

The definitions below concerning infinitesimals follow [10] (see also [7]).

Let $\mathbf{F}$ be an arbitrary real closed field (see, e.g., [14]) and let an element $\varepsilon$ be infinitesimal relative to elements of $\mathbf{F}$. The latter means that for any positive element $a \in \mathbf{F}$ inequalities $0<\varepsilon<a$ are valid in the ordered field $\mathbf{F}(\varepsilon)$. Obviously, the element $\varepsilon$ is transcendental over $\mathbf{F}$. For an ordered field $\mathbf{F}^{\prime}$ we denote by $\tilde{\mathbf{F}}^{\prime}$ its (unique up to isomorphism) real closure, preserving the order on $\mathbf{F}^{\prime}$ [14].

We remember some other well-known statements concerning real closed fields. A Puiseux (formal power-fractional) series over $\mathbf{F}$ is a series of the kind

$$
b=\sum_{i \geq 0} a_{i} \varepsilon^{\nu_{i} / \mu}
$$

where $0 \neq a_{i} \in \mathbf{F}$ for all $i \geq 0$, integers $\nu_{0}<\nu_{1}<\cdots$ increase, and the natural number $\mu \geq 1$. The field $\mathbf{F}\left(\left(\varepsilon^{1 / \infty}\right)\right.$ ) consisting of all Puiseux series (appended by zero) is real closed, hence $\mathbf{F}\left(\left(\varepsilon^{1 / \infty}\right)\right) \supset \widetilde{\mathbf{F}(\varepsilon)} \supset \mathbf{F}(\varepsilon)$. Besides the field $\mathbf{F}[\sqrt{-1}]\left(\left(\varepsilon^{1 / \infty}\right)\right)$ is algebraically closed.

If $\nu_{0}<0$, then the element $b \in \mathbf{F}\left(\left(\varepsilon^{1 / \infty}\right)\right)$ is infinitely large. If $\nu_{0}>0$, then $b$ is infinitesimal relative to elements of the field $\mathbf{F}$. A vector $\left(b_{1}, \ldots, b_{n}\right) \in\left(\mathbf{F}\left(\left(\varepsilon^{1 / \infty}\right)\right)\right)^{n}$ is 
called $\mathbf{F}$-finite if each coordinate $b_{i}, 1 \leq i \leq n$, is not infinitely large relative to elements of $\mathbf{F}$.

For any $\mathbf{F}$-finite element $b \in \mathbf{F}\left(\left(\varepsilon^{1 / \infty}\right)\right)$ its standard part st $(b)$ is definable, namely st $(b)=a_{0}$ in the case $v_{0}=0$ and $\operatorname{st}(b)=0$ if $\nu_{0}>0$. For any F-finite vector $\left(b_{1}, \ldots, b_{n}\right) \in\left(\mathbf{F}\left(\left(\varepsilon^{1 / \infty}\right)\right)\right)^{n}$ its standard part is defined by the equality

$$
\mathrm{st}\left(b_{1}, \ldots, b_{n}\right)=\left(\mathrm{st}\left(b_{1}\right), \ldots, \mathrm{st}\left(b_{n}\right)\right) .
$$

For a set $\mathcal{W} \subset\left(\mathbf{F}\left(\left(\varepsilon^{1 / \infty}\right)\right)\right)^{n}$ we define

$$
\operatorname{st}(\mathcal{W})=\{\operatorname{st}(w): w \in \mathcal{W} \text { and } w \text { is } \mathbf{F} \text {-finite }\} .
$$

The following "transfer principle" is true [21]. If $\mathbf{F}^{\prime}, \mathbf{F}^{\prime \prime}$ are real closed fields with $\mathbf{F}^{\prime} \subset \mathbf{F}^{\prime \prime}$ and $\mathcal{P}$ is a closed (without free variables) formula of the first-order theory of the field $\mathbf{F}^{\prime}$, then $\mathcal{P}$ is true over $\mathbf{F}^{\prime}$ if and only if $\mathcal{P}$ is true over $\mathbf{F}^{\prime \prime}$.

In what follows we consider infinitesimals $\varepsilon_{1}, \varepsilon_{2}, \ldots$ such that $\varepsilon_{i+1}$ is infinitesimal relative to the real closure $\mathbf{R}_{i}$ of the field $\mathbf{R}\left(\varepsilon_{1}, \ldots, \varepsilon_{i}\right)$ for each $i \geq 0$. We assume that $\mathbf{R}_{0}=\mathbf{R}$.

For an $\mathbf{R}_{i}$-finite element $b \in \mathbf{R}_{i+1}$ its standard part (relative to $\mathbf{R}_{i}$ ) is denoted by $\mathrm{st}_{i}(b) \in \mathbf{R}_{i}$. For any $b \in \mathbf{R}_{j}, j>i$, we define st $(b)=\mathrm{st}_{i}\left(\mathrm{st}_{i+1}\left(\cdots \mathrm{st}_{j-1}(b) \cdots\right)\right.$. For a semialgebraic set $V \subset \mathbf{F}_{1}^{n}$ defined by a certain formula $\Phi$ of the first-order theory of the real closed field $\mathbf{F}_{1}$ and for a real closed field $\mathbf{F}_{2} \supset \mathbf{F}_{1}$, we define the completion $V^{\left(\mathbf{F}_{2}\right)} \subset \mathbf{F}_{2}^{n}$ of $V$ as the semialgebraic set given in $\mathbf{F}_{2}^{n}$ by the same formula $\Phi$ (we say that $V^{\left(\mathbf{F}_{2}\right)}$ is defined over $\left.\mathbf{F}_{1}\right)$. We omit superindex $\left(\mathbf{F}_{2}\right)$ in $V^{\left(\mathbf{F}_{2}\right)}$ when this does not lead to ambiguity. Completions of polynomials and algebraic functions can be defined in a similar way.

For any quadratic-irrational function (see Section 1) of the form

$$
f\left(X_{1}, \ldots, X_{n}, \sqrt{\varphi_{1}}, \ldots, \sqrt{\varphi_{r}}\right),
$$

where

$$
f \in \mathbf{R}_{i}\left[X_{1}, \ldots, X_{n}, Z_{1}, \ldots, Z_{r}\right], \quad \varphi_{e} \in \mathbf{R}_{i}\left[X_{1}, \ldots, X_{n}\right], \quad 1 \leq e \leq r,
$$

and a point $y \in \mathbf{R}_{j}^{n}, j \geq i$, such that $\mathrm{st}_{i}(y)$ is definable and $\varphi_{e}(y) \geq 0,1 \leq e \leq r$, we have $\mathrm{st}_{i}(f(y))=f\left(\mathrm{st}_{i}(y)\right)$. Indeed, for Puiseux series $b$ (see above) with $b>0$ (and therefore with $a_{0}>0$ ) its square root

$$
\sqrt{b}=\sqrt{a_{0}} \varepsilon^{\nu_{0} /(2 \mu)}+\cdots .
$$

Hence $\operatorname{st}(\sqrt{b})=\sqrt{\operatorname{st}(b)}$ is 0 if $\nu_{0}>0$ and equals $\sqrt{a_{0}}$ if $\nu_{0}=0$.

Note that the transfer principle can also be applied to a formula containing quadraticirrational functions, since any such formula can be replaced by an equivalent formula of first-order theory. This can be done by replacing each occurrence of $\sqrt{\varphi_{e}}$ with a new variable $Z_{e}$, adding the quantifier prefix $\exists Z_{e}$ and inequalities $Z_{e} \geq 0, Z_{e}^{2}=\varphi_{e}, 1 \leq$ $e \leq r$.

Denote by $B_{x}(r)$ the open ball in $\mathbf{R}_{i}^{n}$ centered at $x$ and of radius $r$, and denote by $\|\cdot\|$ the completion of the Euclidean distance function.

The following lemma shows that the standard part of a semialgebraic set coincides with the standard part of its completion. 
Lemma 2. Let $\mathbf{R}_{m} \subset \mathbf{F} \subset \mathbf{R}_{j}$ where $\mathbf{F}$ is a real closed field and $V \subset \mathbf{F}^{n}$ is a semialgebraic set defined over $\mathbf{F}$. Then $\mathrm{st}_{m}(V)=\mathrm{st}_{m}\left(V^{\left(\mathbf{R}_{j}\right)}\right)$.

Proof. The inclusion

$$
\mathrm{st}_{m}(V) \subset \mathrm{st}_{m}\left(V^{\left(\mathbf{R}_{j}\right)}\right)
$$

is trivial.

To prove the opposite inclusion take a point $x \in \mathrm{st}_{m}\left(V^{\left(\mathbf{R}_{j}\right)}\right)$ and consider a semialgebraic set $\left\{\|x-y\|^{2}: y \in V\right\} \subset \mathbf{F}$. This set is a finite union of (either closed, open, or semiopen) intervals. This is obvious for a semialgebraic subset of $\mathbf{R}$, and for an arbitrary real closed field this follows from the transfer principle. Let $\omega$ be the left endpoint of the leftmost among these intervals. If $x \notin \mathrm{st}_{m}(V)$, then there exists $0<r_{0} \in \mathbf{R}_{m}$ such that $\omega>r_{0}^{2}$, hence $B_{x}\left(r_{0}\right) \cap V=\emptyset$. By the transfer principle the completion of the latter set is also empty: $B_{x}\left(r_{0}\right) \cap V^{\left(\mathbf{R}_{j}\right)}=\emptyset$. This contradicts the inclusion $x \in \mathrm{st}_{m}\left(V^{\left(\mathbf{R}_{j}\right)}\right)$ and proves the lemma.

For a subset $E \subset \mathbf{R}_{m}^{n}$ denote by $\operatorname{cl}(E)$ its closure in the topology with the base of all open balls. Denote by $\partial E$ the boundary

$$
\left\{y \in \mathbf{R}_{m}^{n} \text { : for any } 0<r \in \mathbf{R}_{m}, \emptyset \neq B_{y}(r) \cap E \neq B_{y}(r)\right\} .
$$

Note that the above definition of the closure, being applied to a semialgebraic set and written as a formula of first-order theory of the field $\mathbf{R}_{m}$, involves quantifiers. The following lemma shows that the closure of a semialgebraic set can be described in terms of infinitesimals.

Lemma 3 (see Lemma 1 of [11]).

(a) Let polynomials $\varphi_{e} \in \mathbf{R}_{q}\left[X_{1}, \ldots X_{n}\right], 1 \leq e \leq r$, and quadratic-irrational functions

$$
h_{1}, \ldots, h_{j}, g_{1}, \ldots, g_{s} \in \mathbf{R}_{q}\left[X_{1}, \ldots, X_{n}, \sqrt{\varphi_{1}}, \ldots, \sqrt{\varphi_{r}}\right]
$$

and natural numbers $q, l, m$ satisfy inequalities $q<l<m$. Consider the semialgebraic sets

$$
\begin{aligned}
& V=\left\{\varphi_{1}>0, \ldots, \varphi_{r}>0, g_{1} \geq 0, \ldots, g_{s} \geq 0, h_{1}>0, \ldots, h_{j}>0\right\} \subset \mathbf{R}_{q}^{n} \\
& \text { and } \\
& \mathcal{V}=\left\{\varphi_{1}>\varepsilon_{l}, \ldots, \varphi_{r}>\varepsilon_{l}, g_{1}>-\varepsilon_{m}, \ldots, g_{s}>-\varepsilon_{m}, h_{1}>\varepsilon_{l}, \ldots, h_{j}>\varepsilon_{l}\right\} \\
& \subset \mathbf{R}_{m}^{n} \text {. }
\end{aligned}
$$

Then

$$
\operatorname{cl}(V)=\mathrm{st}_{q}(\mathcal{V})=\mathrm{st}_{q}(\mathrm{cl}(\mathcal{V}))
$$

(b) $\partial V \subset \mathrm{st}_{q}(\partial \mathcal{V})$. 
Proof. (a) Let $x \in \operatorname{cl}(\mathcal{V})$ and the standard part $y=\mathrm{st}_{q}(x)$ be definable. We prove that $y \in \operatorname{cl}(V)$. Consider a point $y_{1}=\operatorname{st}_{l}(x)$, then

$$
\begin{gathered}
\varphi_{e}\left(y_{1}\right)=\operatorname{st}_{l}\left(\varphi_{e}(x)\right) \geq \varepsilon_{l}, \quad 1 \leq e \leq r ; \quad g_{s_{1}}\left(y_{1}\right)=\operatorname{st}_{l}\left(g_{s_{1}}(x)\right) \geq 0, \quad 1 \leq s_{1} \leq s \\
h_{j_{1}}\left(y_{1}\right)=\operatorname{st}_{l}\left(h_{j_{1}}(x)\right) \geq \varepsilon_{l}, \quad 1 \leq j_{1} \leq j .
\end{gathered}
$$

Hence $y_{1} \in V^{\left(\mathbf{R}_{i}\right)}$.

If $y \notin \operatorname{cl}(V)$, then there exists $0<r \in \mathbf{R}_{q}$ such that $B_{y}(r) \cap V=\emptyset$. Due to the transfer principle the latter relation also holds over the field $\mathbf{R}_{l}$, namely, $B_{y}(r) \cap V^{\left(\mathbf{R}_{l}\right)}=\emptyset$. On the other hand, $y_{1} \in B_{y}(r) \cap V^{\left(R_{l}\right)}$ since $s_{q}\left(y_{1}\right)=y$. The contradiction obtained proves the inclusion $\operatorname{st}_{q}(\operatorname{cl}(\mathcal{V})) \subset \operatorname{cl}(V)$.

Now let $y \in \operatorname{cl}(V)$. Consider a semialgebraic set $\left\{\|y-z\|^{2}: z \in \mathcal{V}\right\} \subset \mathbf{R}_{m}$. Then this set is a finite union of (either closed, open, or semiopen) intervals (see the proof of Lemma 2). Let $\omega$ be the left endpoint of the leftmost among these intervals. If $y \notin \mathrm{st}_{q}(\mathcal{V})$, then there exists an element $r_{1}, 0<r_{1} \in \mathbf{R}_{q}$, such that $\omega>r_{1}^{2}$, i.e., $\mathcal{V} \cap B_{y}\left(r_{1}\right)=\emptyset$. On the other hand, $V \cap B_{y}\left(r_{1}\right) \neq \emptyset$ since $y \in \operatorname{cl}(V)$. Taking into the account the inclusion $V \subset \mathcal{V}$, we get a contradiction which proves the inclusion $\operatorname{cl}(V) \subset \mathrm{st}_{q}(\mathcal{V})$.

(b) Let $x \in \partial V$ and $x \notin \mathrm{st}_{q}(\partial \mathcal{V})$. Then there exists an element $r_{2}, 0<r_{2} \in \mathbf{R}_{q}$, such that $B_{x}\left(r_{2}\right) \cap \partial \mathcal{V}=\emptyset$ (see the proof of (a)). Because of $(\mathrm{a}), x \in \mathrm{st}_{q}(\mathcal{V})$, therefore $B_{x}\left(r_{2}\right) \subset \mathcal{V}$. On the other hand, $\mathcal{V} \cap \mathbf{R}_{q}^{n} \subset V$, hence $B_{x}\left(r_{2}\right) \cap \mathbf{R}_{q}^{n} \subset V$; this contradicts the inclusion $x \in \partial V$.

The lemma is proved.

In the proof of Lemma 3(a) it was actually shown that for any semialgebraic set $U \subset \mathbf{R}_{m}^{n}$ we have st ${ }_{q}(U)=\operatorname{st}_{q}(\operatorname{cl}(U)), q<m$.

Corollary. Using the notations of Lemma 3 let

$$
\begin{aligned}
& V_{0}=\left\{\varphi_{1}>0, \ldots, \varphi_{r}>0, h=0, h_{1}>0, \ldots, h_{j}>0\right\} \subset \mathbf{R}_{q}^{n} \\
& \mathcal{V}_{0}=\left\{\varphi_{1}>\varepsilon_{l}, \ldots, \varphi_{r}>\varepsilon_{l}, h=\varepsilon_{m}, h_{1}>\varepsilon_{l}, \ldots, h_{j}>\varepsilon_{l}\right\} \subset \mathbf{R}_{m}^{n}
\end{aligned}
$$

Then $\operatorname{st}_{q}\left(\mathcal{V}_{0}\right) \subset \operatorname{cl}\left(V_{0}\right)$.

To prove the corollary, in Lemma 3(a) instead of $\mathcal{V}$ consider a modified set

$$
\left\{\varphi_{1}>\varepsilon_{l}, \ldots, \varphi_{r}>\varepsilon_{l},-2 \varepsilon_{m}<h<2 \varepsilon_{m}, h_{1}>\varepsilon_{l}, \ldots, h_{j}>\varepsilon_{l}\right\} \supset \mathcal{V}_{0}
$$

Lemma 4 (see Lemma 4(a) in [10]). Let $F$ be a smooth algebraic function defined on an open semialgebraic set $U \subset \mathbf{R}_{i}^{n}$ and determined by a polynomial with coefficients from $\mathbf{R}_{i}$. Then $\varepsilon_{i+1}$ is not a critical value of $F$ (i.e., $\operatorname{grad}_{y}(F)$ does not vanish at any point $\left.y \in\left\{F=\varepsilon_{i+1}\right\} \cap U^{\left(\mathbf{R}_{i+1}\right)}\right)$.

Proof. Sard's theorem [13] and the transfer principle imply the finiteness of the set of all critical values of $F$ in $U^{\left(\mathbf{R}_{i+1}\right)}$, moreover this set lies in $\mathbf{R}_{i}$. 


\section{Curved Points}

For any $i$-face $P_{i}$ denote by $\bar{P}_{i}$ the $i$-plane containing $P_{i}$.

First we reduce Theorem 2 to the case of compact $P$. Let $t$ be the minimal dimension of faces of $P$ and let $P_{t}$ be a face with $\operatorname{dim}\left(P_{t}\right)=t$. Then $\bar{P}_{t}$ is a $t$-plane.

For each $i$-face $P_{i}$ of $P$ with $\operatorname{dim}\left(P_{i} \cap W\right)=i$ choose a point $x_{P_{i}} \in\left(P_{i} \cap W\right)$ such that a suitable neighborhood of $x_{P_{i}}$ in $P_{i}$ is contained in $W$.

First consider the case $t \geq 1$. Choose any hyperplane $\Sigma$ transversal to $P_{t}$ such that the points $x_{P_{i}}$ for all $i$-faces $P_{i}$ lie in one of two semispaces of $\mathbf{R}^{n} \backslash \Sigma$, denote this semispace by $\tilde{\Sigma}$. Replace $P$ by $(P \cap \tilde{\Sigma}) \cup \Sigma$, reducing $t$ by one. Continue this process while $t \geq 1$.

Now consider the case $t=0$. Observe that there exists a linear form $L=\tau_{1} X_{1}+\cdots+$ $\tau_{n} X_{n}$ with $\tau_{j} \in \mathbf{R}, 1 \leq j \leq n$, such that for every $\gamma \in \mathbf{R}$ the intersection $\{L+\gamma \geq 0\} \cap P$ is compact. Take $\gamma$ such that $x_{P_{i}} \in P^{\prime}=\{L+\gamma \geq 0\} \cap P$ for all $P_{i}$. The number of all $i$-faces $P_{i}^{\prime}$ of $P^{\prime}$ such that $\operatorname{dim}\left(P_{i}^{\prime} \cap W\right)=i$ is greater than or equal to the number of all $i$-faces $P_{i}$ of $P$ such that $\operatorname{dim}\left(P_{i} \cap W\right)=i$. From now on we assume, without loss of generality, that $P$ is compact.

For an $m$-plane $Q \subset \mathbf{R}_{j}^{n}$ and a point $x \in \mathbf{R}_{j}^{n}$, denote by $Q(x)$ the $m$-plane parallel to $Q$ and containing $x$.

Two planes $Q_{1}, Q_{2}$ of arbitrary dimensions are called transversal if

$$
\operatorname{dim}\left(Q_{1}(0) \cap Q_{2}(0)\right)=\max \left\{0, \operatorname{dim}\left(Q_{1}(0)\right)+\operatorname{dim}\left(Q_{2}(0)\right)-n\right\} .
$$

For every $0 \leq i<n$ choose an $(n-i)$-plane $\Pi_{n-i}$ (defined over $\mathbf{R}$ ) transversal to every face of the polyhedron $P$.

Recall that in the Introduction we have fixed a branch of the tree $T$, and denoted by $f_{i}, 1 \leq i \leq k$, the polynomials attached to vertices along the branch, and assumed that the signs of these polynomials are

$$
f_{1}=\cdots=f_{k_{1}}=0, \quad f_{k_{1}+1}>0, \ldots, f_{k}>0 .
$$

Denote $f=f_{1}^{2}+\cdots+f_{k_{1}}^{2}$. Fix $0 \leq i<n$ and denote by $f^{(x)}$ the restriction of $f$ on $\Pi_{n-i}(x)$ (for $x \in \mathbf{R}_{j}^{n}$ ).

Definition. A point $y \in\left\{f=\varepsilon_{3}\right\}$ is called $i$-curved if $\operatorname{grad}_{y}\left(f^{(y)}-\varepsilon_{3}\right) \neq 0$, all principal curvatures of the variety $\left\{f^{(y)}=\varepsilon_{3}\right\} \subset \Pi_{n-i}(y)$ at $y$ are greater than ${ }^{-1} \varepsilon_{2}^{-1}$, and $f_{k_{1}+1}(y)>\varepsilon_{2}, \ldots, f_{k}(y)>\varepsilon_{2}$.

Remark. We fix an orthogonal basis in $\Pi_{n-i}(0)$ with coordinates belonging to $\mathbf{R}$. Then in the definition we consider curvatures in $\Pi_{n-i}(y)$ with respect to the basis obtained from the one fixed by the shift $Y \longrightarrow Y+y$.

This definition can be considered as a kind of "localization" of the key concept of an angle point from [12].

Denote the set of all $i$-curved points by $\mathcal{K}_{i} \subset \mathbf{R}_{3}^{n}$. Observe that $\mathcal{K}_{i}$ is semialgebraic due to the remark at the end of Section 1. Denote $K_{i}=\mathrm{st}_{0}\left(\mathcal{K}_{i}\right) \subset \mathbf{R}^{n}$, this set is closed semialgebraic by Lemma 5.1 from [20]. The corollary to Lemma 3 implies that $K_{i} \subset \mathrm{cl}(W)$. 
Lemma 5. Suppose that $P_{i}$ is an $i$-face of $P$ such that $\operatorname{dim}\left(W \cap P_{i}\right)=i$, then $W \cap P_{i}$ $\subset K_{i}$.

Proof. Let $x \in W \cap P_{i}$. Then $f_{j}(x)>c, k_{1}+1 \leq j \leq k$, for a certain $0<c \in \mathbf{R}$. Hence there exists $0<r \in \mathbf{R}$ such that for any point $y$ from the open ball $B_{x}(r)$ we have $f_{j}(y)>c, k_{1}+1 \leq j \leq k$. Due to the transfer principle, $f_{j}(y)>c, k_{1}+1 \leq j \leq k$, for any point $y \in B_{x}(r) \cap \mathbf{R}_{3}^{n}$. Observe that $\left\{f=\varepsilon_{3}\right\} \cap \Pi_{n-i}(x)=\left\{f^{(x)}=\varepsilon_{3}\right\}$ is a smooth hypersurface in $\Pi_{n-i}(x)$, because $x \in \mathbf{R}^{n}$ and $\varepsilon_{3}$ is not a critical value of the polynomial $f^{(x)}$ by Lemma 4.

Our purpose is to prove that $x=\mathrm{st}_{2}(y)$ (and a fortiori $x=\mathrm{st}_{0}(y)$ ) for a suitable $y \in$ $\left\{f=\varepsilon_{3}\right\} \cap \Pi_{n-i}(x)$ such that all principal curvatures of the variety $\left\{f=\varepsilon_{3}\right\} \cap \Pi_{n-i}(x)$ at the point $y$ are greater than $\varepsilon_{2}^{-1}$. This would imply Lemma 5 since $\operatorname{grad}_{y}\left(f^{(x)}-\varepsilon_{3}\right) \neq 0$ (see the definition).

The point $x$ is a vertex of the polyhedron $\mathcal{P}=P \cap \Pi_{n-i}(x)$ because $\Pi_{n-i}$ is transversal to $P_{i}$. Note that for each $(n-i-1)$-face of $\mathcal{P}$ the normalized orthogonal vector (in $\Pi_{n-i}(x)$ ) to this face has all coordinates in $\mathbf{R}$. The vertex $x$ belongs to at least $(n-i)$ among $(n-i-1)$-faces of the polyhedron $\mathcal{P}$.

Choose any $(n-i-1)$-faces $T_{1}, \ldots, T_{n-i}$ of this kind. Denote by $\mathcal{T} \subset \Pi_{n-j}(x)$ the closed cone with vertex at $x$, formed by planes $\bar{T}_{1}, \ldots, \bar{T}_{n-i}$ and containing $\mathcal{P}$.

Observe that for any point $y \in \operatorname{cl}\left(B_{x}(r / 2)\right) \cap \mathbf{R}_{3}^{n}$ the inequalities $f_{j}(y)>c, k_{1}+1 \leq$ $j \leq k$, hold, since $\operatorname{cl}\left(B_{x}(r / 2)\right) \subset B_{x}(r)$. Therefore,

$$
\left\{f=0, f_{j}>c, k_{1}+1 \leq j \leq k\right\} \cap \operatorname{cl}\left(B_{x}(r / 2)\right)=\{f=0\} \cap \operatorname{cl}\left(B_{x}(r / 2)\right) .
$$

Denote $D=\mathcal{T} \cap \operatorname{cl}\left(B_{x}(r / 2)\right)$. For any point $z \in\left\{f=\varepsilon_{3}\right\} \cap \Pi_{n-i}(x) \cap \operatorname{cl}\left(B_{x}(r / 2)\right)$ we have $\operatorname{st}_{2}(z) \in\{f=0\} \cap \Pi_{n-i}(x) \cap \operatorname{cl}\left(B_{x}(r / 2)\right)$ (see Section 2). Hence

$$
\mathrm{st}_{2}(z) \in W \cap \Pi_{n-i}(x) \cap \operatorname{cl}\left(B_{x}(r / 2)\right) \subset \mathcal{P} \cap \operatorname{cl}\left(B_{x}(r / 2)\right) \subset D,
$$

in particular, the distance $\rho(z, D)$ from $z$ to $D$ is infinitesimal relative to $\mathbf{R}_{2}$ (the distance from a point to a bounded set closed in the topology with the base of all open balls, does exist because it is true over the field $\mathbf{R}$; over arbitrary real closed fields use the transfer principle).

Since the set $\left\{f=\varepsilon_{3}\right\} \cap \Pi_{n-i}(x) \cap \mathrm{cl}\left(B_{x}(r / 2)\right)$ is bounded and closed in the topology with the base of all open balls, there exists $\rho_{0}=\max \rho(z, D)$ (see the above argument) where $z$ ranges over all points from $\left\{f=\varepsilon_{3}\right\} \cap \Pi_{n-i}(x) \cap \operatorname{cl}\left(B_{x}(r / 2)\right)$, and $\rho_{0}$ is infinitesimal relative to $\mathbf{R}_{2}$.

We shift (in $\Pi_{n-i}$ ) each $(n-i-1)$-dimensional plane among $\bar{T}_{1}, \ldots, \bar{T}_{n-i}$ parallel to itself outward to the cone $\mathcal{T}$ to the distance $\rho_{0}$. The shifted planes form a new closed cone $\mathcal{T}^{\prime}$ with a vertex $x^{\prime}$. Obviously $\mathcal{T} \subset \mathcal{T}^{\prime}$. Observe that the distance $\left\|x-x^{\prime}\right\|$ is infinitesimal relative to $\mathbf{R}_{2}$. Denote $D^{\prime}=\mathcal{T}^{\prime} \cap \operatorname{cl}\left(B_{x}(r / 2)\right)$. Then $\left\{f=\varepsilon_{3}\right\} \cap \Pi_{n-i}(x) \cap \operatorname{cl}\left(B_{x}(r / 2)\right)$ $\subset D^{\prime}$.

In the plane $\Pi_{n-i}(x)$ choose a hyperplane $Q$ such that the coordinates of the normalized vector orthogonal to $Q$ belong to $\mathbf{R}$, and $\mathcal{T}^{\prime} \cap Q\left(x^{\prime}\right)=\left\{x^{\prime}\right\}$. Take a hyperplane $Q(y)$ (in $\Pi_{n-i}(x)$ ) such that its distance from the point $x^{\prime}$ is positive, belongs to $\mathbf{R}$, and $\mathcal{T}^{\prime} \cap Q(y) \neq \emptyset$. Observe that $\mathcal{T}^{\prime} \cap Q(y)$ is contained in a certain $(n-i-1)$-dimensional 
open ball $B \subset Q(y)$ with the center $z$ such that the radius and the coordinates of the point $z-x^{\prime}$ belong to $\mathbf{R}$.

There exists the unique $(n-i-1)$-dimensional sphere $\mathcal{S} \subset \Pi_{n-i}(x)$ containing both the point $\left(x^{\prime}+z\right) / 2$ and the $(n-i-2)$-dimensional sphere $\partial B$. Then the point $x^{\prime}$ lies outside the $(n-i)$-dimensional open ball $\mathcal{B}$ bounded by $\mathcal{S}$.

Denote by $\mathcal{T}^{\prime \prime} \subset \Pi_{n-i}(x)$ the closed cone with the vertex at $x^{\prime}$ and with the base $\mathcal{S}$. Then $\mathcal{T}^{\prime} \subset\left(\mathcal{T}^{\prime \prime} \backslash \partial \mathcal{T}^{\prime \prime}\right)$ because $\mathcal{T}^{\prime} \cap Q(y) \subset B \subset \mathcal{B}$.

The intersection $\mathcal{S} \cap \partial \mathcal{T}^{\prime \prime}$ is an $(n-i-2)$-dimensional sphere situated in a certain hyperplane $\Gamma$ (in $\Pi_{n-i}(x)$ ). Then $\mathcal{S} \cap \partial \mathcal{T}^{\prime \prime}$ divides $\mathcal{S} \backslash\left(\mathcal{S} \cap \partial \mathcal{T}^{\prime \prime}\right.$ ) into two connected components $\mathcal{S}_{1}$ and $\mathcal{S}_{2}$. Let $\mathcal{S}_{1}$ be located in the same half-space (in $\Pi_{n-i}(x)$ ) with the boundary $\Gamma$ as the point $x^{\prime}$.

Denote by $\mathcal{S}_{1}(\mu)$ the dilation of $\mathcal{S}_{1}$ with the coefficient $\mu$ with respect to the point $x^{\prime}$. Observe that the open cone $\mathcal{T}^{\prime \prime} \backslash \partial \mathcal{T}^{\prime \prime}$ is the disjoint union of the dilations $\mathcal{S}_{1}(\mu)$ over all $0<\mu \in \mathbf{R}_{3}$. There exists the minimal $\mu_{0}>0$ such that

$$
\mathcal{S}_{1}\left(\mu_{0}\right) \cap\left\{f=\varepsilon_{3}\right\} \cap \Pi_{n-i}(x) \cap \operatorname{cl}\left(B_{x}(r / 2)\right) \neq \emptyset .
$$

Then $\mathcal{S}_{1}\left(\mu_{0}\right)$ divides the open cone $\mathcal{T}^{\prime \prime} \backslash \partial \mathcal{T}^{\prime \prime}$ into two connected components, moreover, the set

$$
\left\{f=\varepsilon_{3}\right\} \cap \Pi_{n-i}(x) \cap \operatorname{cl}\left(B_{x}(r / 2)\right)
$$

and all points from $\mathcal{T}^{\prime \prime} \backslash \partial \mathcal{T}^{\prime \prime}$ sufficiently close to the point $x^{\prime}$ belong to the different connected components.

Taking into the account that $f(x)=0$, and applying Lemma 3 from [10] to the polynomial $f^{(x)}$, we conclude that there exists a point $y_{0} \in\left\{f=\varepsilon_{3}\right\} \cap \Pi_{n-i}(x)$ such that the distance $\left\|x-y_{0}\right\|$ is infinitesimal relative to $\mathbf{R}_{2}$. Evidently, $y_{0} \in \operatorname{cl}\left(B_{x}(r / 2)\right)$ and $\left\|y_{0}-x^{\prime}\right\|$ is also infinitesimal relative to $\mathbf{R}_{2}$. Hence $\mu_{0}$ is infinitesimal relative to $\mathbf{R}_{2}$ as well. Therefore, the radius $v$ of the sphere $\mathcal{S}\left(\mu_{0}\right)$ is also infinitesimal relative to $\mathbf{R}_{2}$.

Consider a point

$$
y \in \mathcal{S}_{1}\left(\mu_{0}\right) \cap\left\{f=\varepsilon_{3}\right\} \cap \Pi_{n-i}(x) \cap \operatorname{cl}\left(B_{x}(r / 2)\right) .
$$

Then $\left\|y-x^{\prime}\right\|$ is infinitesimal relative to $\mathbf{R}_{2}$. Besides, the hypersurfaces $\mathcal{S}_{1}\left(\mu_{0}\right)$ and $\left\{f=\varepsilon_{3}\right\} \cap \Pi_{n-i}(x)$ (as well as the set $\left.\left\{f=\varepsilon_{3}\right\} \cap \Pi_{n-i}(x) \cap \operatorname{cl}\left(B_{x}(r / 2)\right)\right)$ have the same tangent plane $T$ (in $\Pi_{n-i}(x)$ ) at the point $y$.

Let $\mathcal{H}_{y}$ be the $(n-i-1) \times(n-i-1)$ matrix introduced in Section 1 (with $f^{(x)}$ playing the role of $F$ and $y$ playing the role of $x$ ). For any normalized vector $v \in T$ the second derivative $v \mathcal{H}_{y} v$ of the function $H_{y}$ (see Section 1) in the direction $v$ is greater than or equal to the corresponding second derivative for the sphere $\mathcal{S}\left(\mu_{0}\right)$ (at the point $y$ ). The latter second derivative equals $1 / v$ (see the proof of Theorem 4 in Chapter 12 of [22]). In particular, for the principal curvatures of the hypersurface $\left\{f^{(x)}=\varepsilon_{3}\right\}=\{f=$ $\left.\varepsilon_{3}\right\} \cap \Pi_{n-i}(x)$ (in $\Pi_{n-i}(x)$ ), the inequalities $\lambda_{2} \geq 1 / \nu, \ldots, \lambda_{n-i} \geq 1 / v$ are valid, hence $\lambda_{2}>\varepsilon_{2}^{-1}, \ldots, \lambda_{n-i}>\varepsilon_{2}^{-1}$.

Thus, the point $y$ is $i$-curved (recall that $f_{j}(y)>c>\varepsilon_{2}, k_{1}+1 \leq j \leq k$, since $\left.y \in B_{x}(r)\right)$.

Finally, $\mathrm{st}_{2}(y)=x$, because $\|x-y\|$ is infinitesimal relative to $\mathbf{R}_{2}$ and $x \in \mathbf{R}^{n}, a$ fortiori $\operatorname{st}_{0}(y)=x$, i.e., $x \in K_{i}$. The lemma is proved. 
Corollary. If $\operatorname{dim}\left(W \cap P_{i}\right)=i$, then $\operatorname{dim}\left(K_{i} \cap P_{i}\right)=i$.

This corollary implies that in order to prove Theorem 2 it is sufficient to bound the number of $i$-faces $P_{i}$ for which $\operatorname{dim}\left(K_{i} \cap P_{i}\right)=i$.

Lemma 6. For any smooth point $z \in K_{i}$ with the dimension $\operatorname{dim}_{z}\left(K_{i}\right) \geq i+1$ the tangent plane $T_{z}$ to $K_{i}$ at $z$ is not transversal to $\Pi_{n-i}$.

Remark. In the particular case $i=0$, Lemma 6 states that $K_{0}$ consists of a finite number of points.

Proof of Lemma 6. First we reduce the proof to the case $i=0$ (so assume in the reduction that $\left.\operatorname{dim}\left(K_{0}\right) \leq 0\right)$. Thus, let $i \geq 1$ and suppose that $e=\operatorname{dim}_{z}\left(K_{i}\right) \geq i+1$. Assume that $T_{z}$ is transversal to $\Pi_{n-i}$, then $\operatorname{dim}\left(T_{z} \cap \Pi_{n-i}(z)\right)=e-i$. Take any $(n-e)$-plane $R \subset \Pi_{n-i}(z)$ defined over $\mathbf{R}$ for which $T_{z} \cap R=\{z\}$. Consider the linear orthogonal projection $\pi: \mathbf{R}_{3}^{n} \rightarrow \mathbf{R}_{3}^{e}$ onto the $e$-subspace along $R$. Then $\operatorname{dim}\left(\pi\left(T_{z}\right)\right)=e$. Therefore, by the implicit function theorem, $\pi\left(K_{i}\right) \subset \mathbf{R}^{e}$ contains the $e$-dimensional ball $B_{\pi(z)}(r)$ for a certain $0<r \in \mathbf{R}$.

For any point $x \in K_{i}$ there is a point $x^{\prime} \in \mathcal{K}_{i}$ such that $\mathrm{st}_{0}\left(x^{\prime}\right)=x$, hence $\mathrm{st}_{0}\left(\pi\left(\mathcal{K}_{i}\right)\right) \supset$ $B_{\pi(z)}(r)$.

For any point $y \in \mathbf{R}^{n}$ the set $\mathcal{K}_{0}^{(y)}$ of 0 -curved points of the restriction $f^{(y)}$ coincides with $\Pi_{n-i}(y) \cap \mathcal{K}_{i}$ according to the definition. Applying the assumption that the lemma is valid for $i=0$ to the polynomial $f^{(y)}$ we obtain the inequality $\operatorname{dim}\left(\operatorname{st}_{0}\left(\Pi_{n-i}(y) \cap \mathcal{K}_{i}\right)\right) \leq$ 0 (taking into the account that $f^{(y)}$ is defined over $\mathbf{R}$ ).

We show that $\pi\left(\mathcal{K}_{i}\right)$ does not contain a ball $B_{w}\left(r_{1}\right)$ for any $0<r_{1} \in \mathbf{R}$ and $w \in \mathbf{R}_{3}^{n}$. Assume the contrary, then there exists a point $w_{1} \in B_{w}\left(r_{1}\right) \cap \mathbf{R}^{e}$. Let $y_{1} \in \mathbf{R}^{n}$ be a point such that $\pi\left(y_{1}\right)=w_{1}$. Denote $\Pi^{\prime}=\pi\left(\Pi_{n-i}\right)$, then $\operatorname{dim}\left(\Pi^{\prime}\right)=e-i, \Pi_{n-i}=\pi^{-1}\left(\Pi^{\prime}\right)$. Then the following inequalities hold:

$$
\operatorname{dim} \operatorname{st}_{0}\left(\Pi^{\prime}\left(w_{1}\right) \cap \pi\left(\mathcal{K}_{i}\right)\right) \geq \operatorname{dimst}\left(\Pi^{\prime}\left(w_{1}\right) \cap B_{w}\left(r_{1}\right)\right)=e-i \geq 1 .
$$

On the other hand, $\Pi^{\prime}\left(w_{1}\right) \cap \pi\left(\mathcal{K}_{i}\right)=\pi\left(\mathcal{K}_{i} \cap \Pi_{n-i}\left(y_{1}\right)\right)$, and, therefore,

$$
\operatorname{dim} \operatorname{st}_{0}\left(\Pi^{\prime}\left(w_{1}\right) \cap \pi\left(\mathcal{K}_{i}\right)\right) \leq \operatorname{dimst}_{0}\left(\mathcal{K}_{i} \cap \Pi_{n-i}\left(y_{1}\right)\right) \leq 0
$$

(the latter inequality was proved above). The contradiction obtained shows that $\pi\left(\mathcal{K}_{i}\right)$ does not contain a ball $B_{w}\left(r_{1}\right)$ for any $0<r_{1} \in \mathbf{R}$.

We claim that, for any ball $B_{y_{2}}\left(r_{2}\right) \subset B_{\pi(z)}(r)$ defined over $\mathbf{R}_{3}$ such that $0<r_{2} \in \mathbf{R}$, the intersection $B_{y_{2}}\left(r_{2}\right) \cap \partial \pi\left(\mathcal{K}_{i}\right) \neq \emptyset$. Assume the contrary. Then either $B_{y_{2}}\left(r_{2}\right) \subset \pi\left(\mathcal{K}_{i}\right)$ or $B_{y_{2}}\left(r_{2}\right) \cap \pi\left(\mathcal{K}_{i}\right)=\emptyset$. The inclusion $B_{y_{2}}\left(r_{2}\right) \subset \pi\left(\mathcal{K}_{i}\right)$ is impossible as was shown above. If $B_{y_{2}}\left(r_{2}\right) \cap \pi\left(\mathcal{K}_{i}\right)=\emptyset$, then st $\operatorname{st}_{0}\left(y_{2}\right) \notin$ sto $_{0}\left(\pi\left(\mathcal{K}_{i}\right)\right)$; the latter contradicts the inclusions $B_{\text {sto }\left(y_{2}\right)}\left(r_{2} / 2\right) \subset B_{\pi(z)}(r) \subset \operatorname{st}_{0}\left(\pi\left(\mathcal{K}_{i}\right)\right)$ of the sets in the space $\mathbf{R}^{e}$. This proves the claim.

Observe that $\operatorname{dim}\left(\partial\left(\pi\left(\mathcal{K}_{i}\right)\right)\right) \leq e-1$. Applying Lemma 5.1 from [20], we get

$$
\operatorname{dim} \operatorname{st}_{0}\left(\partial\left(\pi\left(\mathcal{K}_{i}\right)\right)\right) \leq e-1
$$


On the other hand we now prove that $\operatorname{st}_{0}\left(\partial\left(\pi\left(\mathcal{K}_{i}\right)\right)\right) \supset B_{\pi(z)}(r)$. This contradiction would complete the proof of the reduction of the lemma to the case $i=0$. Indeed, let $y_{3} \in B_{\pi(z)}(r)$. Observe that the set $\left\{\left\|y-y_{3}\right\|^{2}: y \in \partial\left(\pi\left(\mathcal{K}_{i}\right)\right)\right\}$ is semialgebraic. Hence, this set is a finite union of points and intervals (see the proof of Lemma 2). Let $\omega$ be the minimal among these points and the endpoints of these intervals. Suppose that $y_{3} \notin \operatorname{st}_{0}\left(\partial\left(\pi\left(\mathcal{K}_{i}\right)\right)\right)$, i.e., there does not exist $y \in \partial\left(\pi\left(\mathcal{K}_{i}\right)\right)$ such that st $t_{0}(y)=y_{3}$. Thus, $\omega>r_{3}^{2}$ for a suitable $0<r_{3} \in \mathbf{R}$. It follows that $B_{y_{3}}\left(r_{3}\right) \cap \partial\left(\pi\left(\mathcal{K}_{i}\right)\right)=\emptyset$. We get a contradiction with the claim proved above.

Now let $i=0$. Suppose that the statement of the lemma is wrong and $\operatorname{dim}\left(K_{0}\right)=$ $s \geq 1$. There is a linear projection $\phi: \mathbf{R}_{3}^{n} \rightarrow \mathbf{R}_{3}$ onto one of the coordinates such that $\phi\left(K_{0}\right) \supset\left[\eta_{1}^{\prime}, \eta_{2}^{\prime}\right]$ for some $\eta_{1}^{\prime}, \eta_{2}^{\prime} \in \mathbf{R}, \eta_{1}^{\prime}<\eta_{2}^{\prime}$. Since $\operatorname{st}_{0}\left(\varphi\left(\mathcal{K}_{0}\right)\right) \supset\left[\eta_{1}^{\prime}, \eta_{2}^{\prime}\right]$ and $\phi\left(\mathcal{K}_{0}\right) \subset \mathbf{R}_{3}$, being a semialgebraic set, consists of a finite union of intervals and points, there exist $\eta_{1}, \eta_{2} \in \mathbf{R}, \eta_{1}<\eta_{2}$, such that $\phi\left(\mathcal{K}_{0}\right) \supset\left[\eta_{1}, \eta_{2}\right]^{\left(\mathbf{R}_{3}\right)}$.

Our nearest goal is to prove the existence of a semialgebraic curve $C^{\prime} \subset \mathcal{K}_{0}$ such that the mapping $\phi: C^{\prime} \rightarrow\left[\eta_{1}, \eta_{2}\right]^{\left(\mathbf{R}_{3}\right)}$ is bijective. For any point $u \in\left[\eta_{1}, \eta_{2}\right]^{\left(\mathbf{R}_{3}\right)}$ take the unique point $v_{u} \in \mathcal{K}_{0}$ such that $\phi\left(v_{u}\right)=u$ according to the following rule.

A projection $\pi_{1}\left(\phi^{-1}(u)\right)$ of $\phi^{-1}(u)$ onto the coordinate $X_{1}$, being a semialgebraic set, is a union of a finite number of points and intervals (with or without endpoints). Let $\mu_{1}, \mu_{2}$ be the endpoints of the leftmost interval.

Consider four cases. In the first case $\mu_{1}, \mu_{2} \in \mathbf{R}_{3}$, then we put $\mu=\left(\mu_{1}+\mu_{2}\right) / 2$. In the second case the interval is given either by inequality $X<\mu_{2}$ or by inequality $X \leq \mu_{2}$, then we put $\mu=\mu_{2}-1$. In the third case the interval is either $\left\{X>\mu_{1}\right\}$ or $\left\{X \geq \mu_{1}\right\}$, then we put $\mu=\mu_{1}+1$. In the last case the interval coincides with the whole $\mathbf{R}_{3}$, then we put $\mu=0$.

Note that $\mu \in \pi_{1}\left(\phi^{-1}(u)\right)$. We fix the first coordinate of the point $v_{u}$ under construction equal to $\mu$.

Consider the projection $\pi_{2}\left(\phi^{-1}(u) \cap\left\{X_{1}=\mu\right\}\right)$ onto the axis $X_{2}$. Continuing in a similar way, after $n$ steps we obtain a point $v_{u}=(\mu, \ldots) \in \phi^{-1}(u)$. We define the semialgebraic curve $C^{\prime}$ to be the set of all the obtained points $v_{u}$ for $u \in\left[\eta_{1}, \eta_{2}\right]^{\left(\mathbf{R}_{3}\right)}$. The curve $C^{\prime}$ has only a finite number of singular points (this is well known for algebraic curves over $\mathbf{R}$; for arbitrary real closed fields we use the transfer principle). The curve $C^{\prime}$ with the singular points deleted is a finite union of smooth connected semialgebraic curves. Take one of these curves $C$ such that $\phi(C) \supset\left[\eta_{3}, \eta_{4}\right]^{\left(\mathbf{R}_{3}\right)}$ for appropriate $\eta_{3}<$ $\eta_{4}, \eta_{3}, \eta_{4} \in \mathbf{R}$.

Since $C \subset \mathcal{K}_{0}$, Theorem 1 from Chapter 9 in [22] implies that, for any point $w \in C$, its curvature $k(w)$ is greater than or equal to the minimum of principal curvatures of the hypersurface $\left\{f=\varepsilon_{3}\right\}$ at this point $w$, hence $k(w)>\varepsilon_{2}^{-1}$ (according to the definition).

Consider the Gauss map $\mathcal{G}: C \longrightarrow \mathcal{S}^{n-1}$ where $\mathcal{S}^{n-1}$ is an $(n-1)$-sphere and for a point $w \in C$ the image $\mathcal{G}(w)$ is the normalized vector tangent to $C$ at $w$.

We prove the following statement:

For any reals $\theta, l$ and any smooth semialgebraic curve $\mathcal{C}$ with the projection on $a$ certain coordinate axis greater than l and with the curvature at each point greater than $\theta$, there exists a hyperplane $\Delta$ such that the semialgebraic set $\Delta \cap \mathcal{S}^{n-1} \cap \mathcal{G}(\mathcal{C})$ has dimension zero and contains at least $\lfloor l \theta / \pi\rfloor$ points. 
To prove this statement for a curve $\mathcal{C}$ defined over $\mathbf{R}$ observe that the length (with multiplicities) of the image $\mathcal{G}(\mathcal{C}) \subset \mathcal{S}^{n-1}$ equals

$$
\int_{w \in \mathcal{C}} k(w) \geq l \theta
$$

(see Chapter 10 in [22]). Observe that the length of a curve $\mathcal{C}_{1} \subset \mathcal{S}^{n-1}$ equals the average (with respect to the uniform Borel measure) number of points of intersection $\mathcal{C}_{1} \cap \mathcal{S}^{n-1} \cap \Delta$ over all hyperplanes $\Delta$, multiplied by $\pi$. This implies the statement for the semialgebraic curves $\mathcal{C}$ defined over $\mathbf{R}$. For curves $\mathcal{C}$ defined over an arbitrary real closed field this statement follows from the transfer principle (applied for fixed $\theta$ and $l$ ).

Applying the statement to the curve $C$ with $l=\eta_{4}-\eta_{3}$ and fixed arbitrary real $\theta$ (taking into the account that for any point $w \in C$ the curvature $k(w)>\varepsilon_{2}^{-1}>\theta$ ), we conclude that there exists a vector $\left(\rho_{1}, \ldots, \rho_{n}\right)$ such that $C$ contains at least $[l \theta / \pi]$ points $w_{1}$ with the tangent vector $t_{w_{1}}$ to $C$ at $w_{1}$ satisfying the linear equation $t_{w_{1}} \cdot\left(\rho_{1}, \ldots, \rho_{n}\right)=0$, and there is a finite number of such points.

The condition $t_{w_{1}} \cdot\left(\rho_{1}, \ldots, \rho_{n}\right)=0$ on a point $w_{1} \in C$ can be formulated as a formula of the first-order theory of real closed fields (for a fixed $\theta$ ). Therefore, there is only a fixed finite number (depending on $C$ ) of such points $w_{1}$, but since an arbitrary $\theta$ can be taken, we get a contradiction.

This implies that $\operatorname{dim}\left(K_{0}\right) \leq 0$ and completes the proof of the lemma.

\section{Faces of $P$ and Whitney Stratification of $K_{i}$}

Recall that $K_{i}$, as any semialgebraic set, admits a Whitney stratification (see, e.g., [6]). Namely, $K_{i}$ can be represented as a disjoint union $K_{i}=\bigcup_{j} S_{j}$ of a finite number of semialgebraic sets, called strata, which are smooth manifolds and such that:

(1) (Frontier condition) $S_{j_{1}} \cap \operatorname{cl}\left(S_{j_{2}}\right) \neq \emptyset$ if and only if $S_{j_{1}} \subset \operatorname{cl}\left(S_{j_{2}}\right)$ (this defines a partial order $S_{j_{1}} \prec S_{j_{2}}$ on the strata).

(2) (Whitney condition A) Let $S_{j_{1}} \subset \operatorname{cl}\left(S_{j_{2}}\right)$ and a sequence of points $y_{m} \in S_{j_{2}}$ tends to a point $y \in S_{j_{1}}$ when $m \rightarrow \infty$. Assume that the sequence of tangent planes $T_{y_{m}}$ to $S_{j_{2}}$ at points $y_{m}$ tends to a certain plane $T$. Then $T_{y} \subset T$ where $T_{y}$ is a tangent plane to $S_{j,}$ at $y$.

Lemma 7. Suppose that $P_{i}$ is an $i$-face of $P$ such that $\operatorname{dim}\left(K_{i} \cap P_{i}\right)=i$. Assume that $S_{j}^{\prime}$ is a connected component of a stratum $S_{j}$ of $K_{i}$ such that $\operatorname{dim}\left(\mathrm{cl}\left(S_{j}^{\prime}\right) \cap K_{i} \cap P_{i}\right)=i$. Then $S_{j}^{\prime} \subset P_{i}$.

Proof. If $\operatorname{dim}\left(S_{j}\right)=i$, then $S_{j}^{\prime} \subset P_{i}$ because $S_{j}^{\prime} \subset K_{i} \subset \operatorname{cl}(W)$ (see the definition of $K_{i}$ in Section 3$)$ and $\operatorname{cl}(W) \subset P$, taking into account that $S_{j}^{\prime}$ is a connected smooth semialgebraic set.

Now let $e=\operatorname{dim} S_{j} \geq i+1$. We can assume without loss of generality that $S_{j}$ is one of the maximal strata (with respect to the partial order $\prec$ ), otherwise take a maximal stratum containing $S_{j}$ in its closure. 
There is a stratum $S_{l}$ such that $\operatorname{dim}\left(S_{l} \cap \operatorname{cl}\left(S_{j}^{\prime}\right) \cap K_{i} \cap P_{i}\right)=i$. Property (1) of Whitney stratification implies that $S_{l} \subset \operatorname{cl}\left(S_{j}\right)$. Take a connected component $S_{l}^{\prime}$ of $S_{l}$ for which $\operatorname{dim}\left(S_{l}^{\prime} \cap \mathrm{cl}\left(S_{j}^{\prime}\right) \cap K_{i} \cap P_{i}\right)=i$. Then $\operatorname{dim}\left(S_{l}^{\prime}\right)=i$, i.e., $\operatorname{dim}\left(S_{l}\right)=i$ because $S_{l}^{\prime}$ is smooth and $S_{l}^{\prime} \subset P$, hence $S_{l}^{\prime} \subset P_{i}$ arguing as above. Let a point $y \in S_{l}^{\prime} \cap \operatorname{cl}\left(S_{j}^{\prime}\right) \cap K_{i} \cap P_{i}$ be such that for a suitable $0<r \in \mathbf{R}$ we have

$$
\left(B_{y}(r) \cap P_{i}\right) \subset\left(S_{l}^{\prime} \cap \mathrm{cl}\left(S_{j}^{\prime}\right) \cap K_{i} \cap P_{i}\right),
$$

then $T_{y}\left(S_{l}^{\prime}\right)=\bar{P}_{i}$.

There exists a converging sequence $y_{m} \rightarrow_{m \rightarrow \infty} y, y_{m} \in S_{j}^{\prime}$, such that the sequence of $e$-dimensional tangent planes $T_{y_{m}}\left(S_{j}^{\prime}\right)$ converges when $m \rightarrow \infty$ to a certain $e$-dimensional plane $\tau$. Due to (2) (Whitney condition A), $\bar{P}_{i} \subset \tau$.

Lemma 6 implies that $T_{y_{m}}\left(S_{j}^{\prime}\right)$ is not transversal to $\Pi_{n-i}$ (taking into account that $y_{m}$ is a smooth point of $K_{i}$ because $S_{j}$ is a maximal stratum). Therefore, $e_{m}^{\prime}=\operatorname{dim}\left(T_{y_{m}}\left(S_{j}^{\prime}\right) \cap\right.$ $\left.\Pi_{n-i}\left(y_{m}\right)\right) \geq e-i+1$. Some subsequence $T_{y_{m_{q}}}\left(S_{j}^{\prime}\right) \cap \Pi_{n-j}\left(y_{m_{q}}\right)$ of planes converges when $q \rightarrow \infty$ to a certain $e^{\prime}$-dimensional plane $\chi \subset \Pi_{n-i}(y)$, where $e^{\prime}=e_{m_{q}}^{\prime} \geq e-i+1$ for any large enough $q$.

Choose a basis $a_{1}, \ldots, a_{i}$ of $i$-plane $\bar{P}_{i}(0)$ and a basis $b_{1}, \ldots, b_{e^{\prime}}$ of $\chi(0)$. Then vectors $a_{1}, \ldots, a_{i}, b_{1}, \ldots, b_{e^{\prime}}$ are linearly independent due to the transversality of $\bar{P}_{i}$ and $\Pi_{n-i}$. For large enough $q_{0}$, for any $q \geq q_{0}$ there exist vectors

$$
a_{1}^{(q)}, \ldots, a_{i}^{(q)}, b_{1}^{(q)}, \ldots, b_{e^{\prime}}^{(q)} \in\left(T_{y_{m_{q}}}\left(S_{j}^{\prime}\right)\right)(0)
$$

situated sufficiently close to vectors $a_{1}, \ldots, a_{i}, b_{1}, \ldots, b_{e^{\prime}}$, respectively, so that the vectors $a_{1}^{(q)}, \ldots, a_{i}^{(q)}, b_{1}^{(q)}, \ldots, b_{e^{\prime}}^{(q)}$ are also linearly independent. Hence $\operatorname{dim}\left(T_{y_{\mathrm{m}_{q}}}\left(S_{j}^{\prime}\right)\right) \geq$ $e^{\prime}+i \geq e+1$. This leads to a contradiction with the equality $\operatorname{dim}\left(S_{j}\right)=e$ and proves the lemma.

Denote $g=f_{k_{1}+1} \cdots f_{k}$. Choose $0<\mu \in \mathbf{R}$ satisfying the following properties:

(a) $\mu$ is less than the absolute values of all critical values of the restrictions of $g$ on $i$-faces $P_{i}$ (note that Sard's theorem implies the finiteness of the number of all critical values, moreover, they all belong to $\mathbf{R}$ due to Lemma 4).

(b) For any $P_{i}$ such that $\operatorname{dim}\left(K_{i} \cap P_{i}\right)=i$, the dimension

$$
\operatorname{dim}\left(\{g=\mu\} \cap \operatorname{cl}\left(S_{j}^{\prime}\right) \cap K_{i} \cap P_{i}\right) \leq i-2
$$

for every connected component $S_{j}^{\prime}$ of a stratum $S_{j}$ such that $S_{j}^{\prime}$ is not contained in $P_{i}$. Observe that due to Lemma 7 there exists at most a finite number of $\mu$ violating this condition because $\operatorname{dim}\left(\operatorname{cl}\left(S_{j}^{\prime}\right) \cap K_{i} \cap P_{i}\right) \leq i-1$, together with (a) this justifies the existence of the required $\mu$.

Denote $K_{i}^{\prime}=K_{i} \cap\{g=\mu\}$.

\section{Lemma 8.}

$$
K_{i}^{\prime}=\operatorname{st}_{0}\left(\mathcal{K}_{i} \cap\left\{|g-\mu|<\varepsilon_{1}\right\}\right)
$$


Proof. We first prove the inclusion $C$.

Denote by $\mathbf{F}$ the real closure of the field $\mathbf{R}\left(\varepsilon_{2}, \varepsilon_{3}\right)$. Since $\mathcal{K}_{i}$ is defined over $\mathbf{F}$ we have $\mathcal{K}_{i}=\left(\mathcal{K}_{i} \cap \mathbf{F}^{n}\right)^{\left(\mathbf{R}_{3}\right)}$. Apply Lemma 2 to the fields $\mathbf{R} \subset \mathbf{F} \subset \mathbf{R}_{3}$ taking the set $\mathcal{K}_{i} \cap \mathbf{F}^{n}$ as $V$. Then $\mathrm{st}_{0}\left(\mathcal{K}_{i} \cap \mathbf{F}^{n}\right)=\mathrm{st}_{0}\left(\mathcal{K}_{i}\right)=K_{i}$.

Let $x \in K_{i}^{\prime}$. It follows that there exists a point $y \in \mathcal{K}_{i} \cap \mathbf{F}^{n}$ such that $\operatorname{st}_{0}(y)=x$. Hence $\operatorname{st}_{0}(g(y))=g\left(\operatorname{st}_{0}(y)\right)=g(x)=\mu$. Then $(g(y)-\mu) \in \mathbf{F}$ is infinitesimal relative to $\mathbf{R}$. Taking into account the representation of $g(y)-\mu$ as a Puiseux series in $\varepsilon_{3}$ with the coefficients being, in their turn, Puiseux series in $\varepsilon_{2}$ (see Section 2), we deduce that $|g(y)-\mu|<\varepsilon_{1}$. Thus $y \in \mathcal{K}_{i} \cap\left\{|g-\mu|<\varepsilon_{1}\right\}$, which proves the inclusion $C$.

To prove the inclusion $\supset$, take a point $x \in \operatorname{st}_{0}\left(\mathcal{K}_{i} \cap\left\{|g-\mu|<\varepsilon_{1}\right\}\right)$. Then, in particular, $x \in \operatorname{st}_{0}\left(\mathcal{K}_{i}\right)=K_{i}$. There exists a point $y \in \mathcal{K}_{i} \cap\left\{|g-\mu|<\varepsilon_{1}\right\}$ such that $\operatorname{st}_{0}(y)=x$. Then $\mu=\operatorname{st}_{0}(g(y))=g\left(\operatorname{st}_{0}(y)\right)=g(x)$. The lemma is proved.

Lemma 9. For an $i$-face $P_{i}$ of $P$, let the dimension $\operatorname{dim}\left(W \cap P_{i}\right)=i$. The following equality of the varieties holds:

$$
K_{i}^{\prime} \cap P_{i}=\{g=\mu\} \cap\left\{f_{k_{1}+1}>0, \ldots, f_{k}>0\right\} \cap P_{i}
$$

and, moreover, this variety is a nonempty smooth compact hypersurface in $\bar{P}_{i}$. Besides,

$$
\operatorname{dim}\left(\operatorname{cl}\left(K_{i}^{\prime} \backslash P_{i}\right) \cap K_{i}^{\prime} \cap P_{i}\right) \leq i-2 .
$$

Proof. First we prove the inclusion

$$
\left(K_{i}^{\prime} \cap P_{i}\right) \supset\{g=\mu\} \cap\left\{f_{k_{1}+1}>0, \ldots, f_{k}>0\right\} \cap P_{i} .
$$

We have

$$
\left\{f_{k_{1}+1}>0, \ldots, f_{k}>0\right\} \cap P_{i}=\left\{f=0, f_{k_{1}+1}>0, \ldots, f_{k}>0\right\} \cap P_{i}=W \cap P_{i}
$$

since $\operatorname{dim}\left(W \cap P_{i}\right)=i$. By Lemma 5 ,

$$
\left(\left\{f=0, f_{k_{1}+1}>0, \ldots, f_{k}>0\right\} \cap P_{i}\right) \subset\left(K_{i} \cap P_{i}\right) .
$$

Intersecting both sides with the variety $\{g=\mu\}$, we obtain the $\supset$ inclusion.

To prove the inclusion $C$, observe that $f_{j}$ is nonnegative everywhere on $K_{i}$ for each $k_{1}+1 \leq j \leq k$ because $K_{i} \subset \mathrm{cl}(W)$ (see Section 3). On the other hand, $f_{j}$ is nonzero everywhere on $K_{i}^{\prime}$ for $k_{1}+1 \leq j \leq k$ since $f_{k_{1}+1} \cdots f_{k}=\mu$. Thus, $K_{i}^{\prime} \subset\left\{f_{k_{1}+1}>\right.$ $\left.0, \ldots, f_{k}>0\right\}$ which proves the $\subset$ inclusion.

Now we prove that $K_{i}^{\prime} \cap P_{i}$ is a nonempty smooth hypersurface in $\bar{P}_{i}$. Observe that $K_{i}^{\prime} \cap P_{i}$ is bounded because $P$ is compact, besides $K_{i}^{\prime} \cap P_{i}$ is closed since its closure

$$
K_{i}^{\prime} \cap \mathrm{cl}\left(P_{i}\right)=K_{i}^{\prime} \cap \bar{P}_{i} \subset\left\{f_{k_{1}+1}>0, \ldots, f_{k}>0\right\} \cap \bar{P}_{i}=W \cap \bar{P}_{i}=W \cap P_{i} \subset P_{i} .
$$

Since $\operatorname{dim}\left(\left\{f_{k_{1}+1}>0, \ldots, f_{k}>0\right\} \cap P_{i}\right)=i$, each connected component of the set $\left\{f_{k_{1}+1}>0, \ldots, f_{k}>0\right\} \cap P_{i}$ contains a connected component of the smooth hypersurface $\{g=\mu\} \cap \bar{P}_{i}$ (in $\bar{P}_{i}$ ) due to Morse theory (see [13]) and in view of (a). Moreover, each connected component of the hypersurface $\{g=\mu\} \cap \bar{P}_{i}$ either lies completely in the set $\left\{f_{k_{1}+1}>0, \ldots, f_{k}>0\right\} \cap P_{i}$ or does not intersect this set. 
Finally, the inequality

$$
\operatorname{dim}\left(\operatorname{cl}\left(K_{i}^{\prime} \backslash P_{i}\right) \cap K_{i}^{\prime} \cap P_{i}\right) \leq i-2
$$

immediately follows from (b). The lemma is proved.

The next section is dedicated to the proof of the following lemma.

Lemma 10. The number of $i$-faces $P_{i}$ of $P$ such that $K_{i}^{\prime} \cap P_{i}$ is a nonempty compact smooth hypersurface in $\bar{P}_{i}$ and

$$
\operatorname{dim}\left(\operatorname{cl}\left(K_{i}^{\prime} \backslash P_{i}\right) \cap K_{i}^{\prime} \cap P_{i}\right) \leq i-2,
$$

does not exceed $(n k d)^{O(n)}$.

Theorem 2 immediately follows from Lemmas 9 and 10.

\section{Extremal Points of a Linear Function on $K_{i}^{\prime}$}

Take a linear form $L=\gamma_{1} X_{1}+\cdots+\gamma_{n} X_{n}$ with generic coefficients $\gamma_{1}, \ldots, \gamma_{n} \in \mathbf{R}$. Fix $P_{i}$ satisfying the conditions of Lemma 10 and denote by $L^{\left(P_{i}\right)}$ the restriction of $L$ on $\bar{P}_{i}$. Then $L^{\left(P_{i}\right)}$ attains its maximal value, say $\theta_{0}^{\left(P_{i}\right)}$, on the compact set $K_{i}^{\prime} \cap P_{i}$. Since $L^{\left(P_{i}\right)}$ is a generic linear form on $\bar{P}_{i}$ as well, the following two properties are fulfilled:

(i) $L^{\left(P_{i}\right)}$ attains the value $\theta_{0}^{\left(P_{i}\right)}$ at a unique point $v \in K_{i}^{\prime} \cap P_{i}$ (see [13]).

(ii) The point $v$ does not belong to $\operatorname{cl}\left(K_{i}^{\prime} \backslash P_{i}\right)$ (see the conditions of Lemma 10).

Indeed, the semialgebric set of linear forms on $\bar{P}_{i}$ for which properties (i) and (ii) fail, has dimension less than the dimension of the set of all linear forms on $\bar{P}_{i}$, and thus for the generic form $L$ properties (i) and (ii) are valid.

Denote by $V$ a connected component of $K_{i}^{\prime} \cap P_{i}$ which contains $v$. Property (ii) implies that there exists $0<r \in \mathbf{R}$ such that $B_{v}(r) \cap K_{i}^{\prime}=B_{v}(r) \cap V$. Thus, $L$ attains a local isolated maximum on $K_{i}^{\prime}$ at the point $v$ by property (i). Hence, there exists an element $0<\zeta^{\left(P_{i}\right)} \in \mathbf{R}$ such that the values of $L$ on the set $\partial B_{v}(r / 2) \cap K_{i}^{\prime}$ are less than $\theta_{0}^{\left(P_{i}\right)}-\zeta^{\left(P_{i}\right)}$

Lemma 11. The linear form $L$ attains its maximal value $\theta^{\left(P_{i}\right)}$ on the set

$$
\operatorname{cl}\left(\mathcal{K}_{i} \cap\left\{|g-\mu|<\varepsilon_{1}\right\}\right) \cap B_{v}(r / 2)
$$

(say, at a point $w$ ) and the values of $L$ on the set

$$
\operatorname{cl}\left(\mathcal{K}_{i} \cap\left\{|g-\mu|<\varepsilon_{1}\right\}\right) \cap \partial B_{v}(r / 2)
$$

are less than $\mathrm{st}_{0}\left(\theta^{\left(P_{i}\right)}\right)-\zeta^{\left(P_{i}\right)}$. Moreover, $\mathrm{st}_{0}\left(\theta^{\left(P_{i}\right)}\right)=\theta_{0}^{\left(P_{i}\right)}$ and $\mathrm{st}_{0}(w)=v \in P_{i}$. 
Proof. Due to Lemma 8 and the remark after Lemma 3 from Section 2, we have

$$
\operatorname{st} \operatorname{cl}_{0}\left(\mathcal{K}_{i} \cap\left\{|g-\mu|<\varepsilon_{1}\right\}\right)=K_{i}^{\prime} .
$$

By the transfer principle, $L$ attains its maximum $\theta^{\left(P_{i}\right)}$ on the closed bounded $\operatorname{set} \operatorname{cl}\left(\mathcal{K}_{i} \cap\right.$ $\left.\left\{|g-\mu|<\varepsilon_{1}\right\}\right) \cap \operatorname{cl}\left(B_{v}(r / 2)\right)$ at some point $w$. Then $\operatorname{st}_{0}\left(\theta^{\left(P_{i}\right)}\right)=\dot{\theta}_{0}^{\left(P_{i}\right)}$ and st $\mathrm{st}_{0}(w)=v$ (due to (i)).

Since

$$
\operatorname{st}_{0}\left(\operatorname{cl}\left(\mathcal{K}_{i} \cap\left\{|g-\mu|<\varepsilon_{1}\right\}\right) \cap \partial B_{v}(r / 2)\right) \subset K_{i}^{\prime} \cap \partial B_{v}(r / 2)
$$

we obtain the second statement of the lemma from the defining property of $\zeta^{\left(P_{i}\right)}$. The lemma is proved.

Lemma 11 states that $L$ attains a local maximum on the $\operatorname{set} \operatorname{cl}\left(\mathcal{K}_{i} \cap\left\{|g-\mu|<\varepsilon_{1}\right\}\right)$ at a point $w$ such that $\operatorname{st}_{0}(w) \in P_{i}$. In order to estimate the number of such local maximum values of $L$ we now construct a smooth hypersurface which is infinitely close to $\operatorname{cl}\left(\mathcal{K}_{i} \cap\left\{|g-\mu|<\varepsilon_{1} \mid\right)\right.$. After that it will be sufficient to bound the number of local maxima of $L$ on this smooth hypersurface.

For a point $y$ denote the coordinates of the gradient

$$
\operatorname{grad}_{y}\left(f^{(y)}-\varepsilon_{3}\right)=\left(u_{1}, \ldots, u_{n-i}\right)
$$

(see the definition). The set $\mathcal{K}_{i} \cap\left\{|g-\mu|<\varepsilon_{1}\right\}$ of the points $y=\left(y_{1}, \ldots, y_{n}\right)$ can be represented as a union of $n-i$ semialgebraic sets of the form

$$
U^{\left(i_{0}\right)}=\left\{f-\varepsilon_{3}=0, u_{i_{0}}^{2}>0, p_{1}>0, \ldots, p_{s}>0\right\} \subset \mathbf{R}_{3}^{n}, \quad 1 \leq i_{0} \leq n-i,
$$

for some algebraic functions $p_{1}, \ldots, p_{s}$ of the quadratic-irrational type introduced in Section 1, i.e., polynomials (with coefficients from $\mathbf{R}_{2}$ ) in $y_{1}, \ldots, y_{n}$ and in

$$
\sqrt{u_{i_{0}}^{2}}, \sqrt{u_{i_{0}}^{2}+u_{\pi_{i_{0}}(2)}^{2}}, \cdots, \sqrt{u_{i_{0}}^{2}+u_{\pi_{i_{0}}(2)}^{2}+\cdots+u_{\pi_{i_{0}}(n-i)}^{2}}
$$

(see Lemma 1). Here $\pi_{i_{0}}$ is a permutation of $\{1,2, \ldots, n-i\}$ such that $\pi_{i_{0}}(1)=i_{0}$ (see Section 1).

Denote

$$
q=\left(\varepsilon_{5}^{2}-\left(f-\varepsilon_{3}\right)^{2}\right)\left(u_{i_{0}}^{2}-\varepsilon_{4}\right)\left(p_{1}-\varepsilon_{4}\right) \cdots\left(p_{s}-\varepsilon_{4}\right) .
$$

Introduce semialgebraic sets

$$
\mathcal{U}_{0}^{\left(i_{0}\right)}=\left\{\varepsilon_{5}^{2}>\left(f-\varepsilon_{3}\right)^{2}, u_{i_{0}}^{2}>\varepsilon_{4}, p_{1}>\varepsilon_{4}, \ldots, p_{s}>\varepsilon_{4}\right\} \subset \mathbf{R}_{5}^{n}
$$

and

$$
\mathcal{U}^{\left(i_{0}\right)}=\left\{q=\varepsilon_{6}\right\} \cap\left(\mathcal{U}_{0}^{\left(i_{0}\right)}\right)^{\left(\mathbf{R}_{6}\right)} \subset \mathbf{R}_{6}^{n} .
$$

Lemma 12 (see Lemmas 1 and 4 in [11]).

$$
\operatorname{st}_{3}\left(\mathcal{U}^{\left(i_{0}\right)}\right)=\operatorname{cl}\left(U^{\left(i_{0}\right)}\right) .
$$


Proof. We first show that to prove the lemma it is sufficient to establish the equality

$$
\mathrm{st}_{5}\left(\mathcal{U}^{\left(i_{0}\right)}\right)=\partial \mathcal{U}_{0}^{\left(i_{0}\right)} .
$$

Indeed, due to Lemma 3(a),

$$
\operatorname{st}_{3}\left(\partial \mathcal{U}_{0}^{\left(i_{0}\right)}\right) \subset \operatorname{st}_{3}\left(\operatorname{cl}\left(\mathcal{U}_{0}^{\left(i_{0}\right)}\right)\right)=\operatorname{cl}\left(U^{\left(i_{0}\right)}\right),
$$

thus, due to (4), st ${ }_{3}\left(\mathcal{U}^{\left(i_{0}\right)}\right) \subset \operatorname{cl}\left(U^{\left(i_{0}\right)}\right)$. On the other hand, $\operatorname{cl}\left(U^{\left(i_{0}\right)}\right)=\partial\left(U^{\left(i_{0}\right)}\right)$ because $U^{\left(i_{0}\right)} \subset\left\{f=\varepsilon_{3}\right\}$ and thereby $U^{\left(i_{0}\right)}$ contains no internal points. Hence, Lemma 3(b) implies that $\operatorname{cl}\left(U^{\left(i_{0}\right)}\right) \subset \mathrm{st}_{3}\left(\partial \mathcal{U}_{0}^{\left(i_{0}\right)}\right)$. It follows from (4) that $\operatorname{cl}\left(U^{\left(i_{0}\right)}\right) \subset \mathrm{st}_{3}\left(\mathcal{U}^{\left(i_{0}\right)}\right)$. This would prove the lemma, provided that (4) holds.

Now we prove (4) starting with the inclusion $C$.

Let a point $y \in \mathcal{U}^{\left(i_{0}\right)}$ and the standard part $x=\operatorname{st}_{5}(y)$ be definable. Then $q(x)=0$ and

$$
\varepsilon_{5}^{2} \geq\left(f-\varepsilon_{3}\right)^{2}, \quad u_{i_{0}}^{2} \geq \varepsilon_{4}, \quad p_{1} \geq \varepsilon_{4}, \ldots, p_{s} \geq \varepsilon_{4} .
$$

Suppose that $x \notin \partial \mathcal{U}_{0}^{\left(i_{0}\right)}$. Therefore there exists $0<r_{0} \in \mathbf{R}_{5}$ such that either $B_{x}\left(r_{0}\right) \subset$ $\mathcal{U}_{0}^{\left(i_{0}\right)}$ or $B_{x}\left(r_{0}\right) \cap \mathcal{U}_{0}^{\left(i_{0}\right)}=\emptyset$.

If $B_{x}\left(r_{0}\right) \subset \mathcal{U}_{0}^{\left(i_{0}\right)}$ we get a contradiction with $q(x)=0$.

If $B_{x}\left(r_{0}\right) \cap \mathcal{U}_{0}^{\left(i_{0}\right)}=\emptyset$ we conclude that the intersection $B_{x}\left(r_{0}\right) \cap\left(\mathcal{U}_{0}^{\left(i_{0}\right)}\right)^{\left(\mathbf{R}_{6}\right)}=\emptyset$. Since $y$ belongs to this intersection we again get a contradiction which proves the inclusion $C$ in (4).

To prove the inclusion $\supset$ in (4) take a point $x \in \partial \mathcal{U}_{0}^{\left(i_{0}\right)}$. Observe that $q$ is positive everywhere on $\mathcal{U}_{0}^{\left(i_{0}\right)}$ and $q$ vanishes everywhere on $\partial \mathcal{U}_{0}^{\left(i_{0}\right)}$, in particular $q(x)=0$.

Suppose that $x \notin \operatorname{st}_{5}\left(\mathcal{U}^{\left(i_{0}\right)}\right)$. Then there exists $0<r_{1} \in \mathbf{R}_{5}$ such that $B_{x}\left(r_{1}\right) \cap \mathcal{U}^{\left(i_{0}\right)}=$ $\emptyset$ (see the proof of Lemma 2). Consider the decomposition of the intersection

$$
B_{x}\left(r_{1}\right) \cap \mathcal{U}_{0}^{\left(i_{0}\right)}=\bigcup_{j} \mathcal{U}_{j}
$$

into its connected components (which are also semialgebraic sets and there is a finite number of them, see, e.g., [10]). Since $x \in \operatorname{cl}\left(B_{x}\left(r_{1}\right) \cap \mathcal{U}_{0}^{\left(i_{0}\right)}\right)$ there is $j_{0}$ for which $x \in \operatorname{cl}\left(\mathcal{U}_{j_{0}}\right)$.

Observe that in the set $\mathcal{U}_{0}^{\left(i_{0}\right)} \subset\left\{u_{i_{0}}^{2}>0\right\}$ all the square roots (3) are positive. Therefore, by the transfer principle, all quadratic-irrational functions $p_{1}, \ldots, p_{s}$ are continuous in $\mathcal{U}_{0}^{\left(i_{0}\right)}$, hence $q$ is continuous. All the values of the continuous function $q$ on $\mathcal{U}_{j_{0}}$ form a connected semialgebraic subset $\Xi \subset \mathbf{R}_{5}$. So, $\Xi$ is an interval (or a point) with endpoints $\xi_{1}, \xi_{2} \in \mathbf{R}_{5}, \xi_{1} \leq \xi_{2}$, and the set $\Xi$ could be either closed, open, or semiopen. Observe that $\xi_{1} \geq 0$ because $\mathcal{U}_{j_{0}} \subset \mathcal{U}_{0}^{\left(i_{0}\right)}$. On the other hand, $\xi_{1}=0$ since $q(x)=0$ and $x \in \operatorname{cl}\left(\mathcal{U}_{j_{0}}\right)$. Obviously, $\xi_{2}>0$. Due to the transfer principle, $q$ attains on the $\operatorname{set} \mathcal{U}_{j_{0}}^{\left(\mathbf{R}_{6}\right)}$ all the values from the interval $\left(0, \xi_{2}\right)^{\left(\mathbf{R}_{6}\right)}$. In particular, there exists a point $y \in \mathcal{U}_{j_{0}}^{\left(\mathbf{R}_{6}\right)}$ such that $q(y)=\varepsilon_{6}$. Then $y \in B_{x}\left(r_{1}\right) \cap \mathcal{U}^{\left(i_{0}\right)}$. The contradiction obtained completes the proof of the lemma.

Lemma 13. For a certain $1 \leq i_{0} \leq n-i$ the linear form $L$ attains its maximal value $\theta_{1}^{\left(P_{i}\right)}$ on the set $\operatorname{cl}\left(\mathcal{U}^{\left(i_{0}\right)}\right) \cap B_{v}(r / 2)$ at a certain point $w_{1}$, and the values of $L$ on the 
set $\mathrm{cl}\left(\mathcal{U}^{\left(i_{0}\right)}\right) \cap \partial B_{v}(r / 2)$ are less than $\mathrm{st}_{0}\left(\theta_{1}^{\left(P_{i}\right)}\right)-\zeta^{\left(P_{i}\right)}$. Moreover, $\mathrm{st}_{3}\left(\theta_{1}^{\left(P_{i}\right)}\right)=\theta^{\left(P_{i}\right)}$ and $\operatorname{st}_{0}\left(w_{1}\right)=v \in P_{i}$.

Proof. Since

$$
\mathcal{K}_{i} \cap\left\{|g-\mu|<\varepsilon_{1}\right\}=\bigcup_{1 \leq i_{0} \leq n-i} U^{\left(i_{0}\right)}
$$

there is $1 \leq i_{0} \leq n-i$ such that $w \in \operatorname{cl}\left(U^{\left(i_{i 1}\right)}\right)$ (see Lemma 11 ). The linear form $L$ attains its maximum $\theta_{1}^{\left(P_{i}\right)}$ on the bounded closed $\operatorname{set} \operatorname{cl}\left(\mathcal{U}^{\left(i_{1}\right)}\right) \cap \mathrm{cl}\left(B_{v}(r / 2)\right)$ at a point $w_{1}$. Using Lemma 12 and the equality st ${ }_{3}\left(\mathcal{U}^{\left(i_{0}\right)}\right)=\operatorname{st}_{3}\left(\operatorname{cl}\left(\mathcal{U}^{\left(i_{0}\right)}\right)\right.$ ) (see the remark in Section 2), we get $\mathrm{st}_{3}\left(\theta_{1}^{\left(P_{i}\right)}\right)=\theta^{\left(P_{i}\right)}$. Due to $(\mathrm{i}), \mathrm{st}_{0}\left(w_{1}\right)=v$.

The values of $L$ on the set $\operatorname{cl}\left(\mathcal{U}^{\left(i_{0}\right)}\right) \cap \partial B_{v}(r / 2)$ are less than $\theta_{0}^{\left(P_{i}\right)}-\zeta^{\left(P_{i}\right)}$ due to the similar statement in Lemma 11 , taking into the account that

$$
\mathrm{st}_{3}\left(\operatorname{cl}\left(\mathcal{U}^{\left(i_{0}\right)}\right) \cap \partial B_{v}(r / 2)\right) \subset \partial B_{v}(r / 2) .
$$

The lemma is proved.

Since $w_{1}$ is a local maximum of $L$ on the $\operatorname{set} \operatorname{cl}\left(\mathcal{U}^{\left(i_{1}\right)}\right)$ we obtain the following corollary.

Corollary. The number of $i$-faces $P_{i}$ satisfying the conditions of Lemma 10 does not exceed the number of all the values of local maxima of the linear form $L$ on the set

$$
\bigcup_{1 \leq i_{0} \leq n-i} \operatorname{cl}\left(\mathcal{U}^{\left(i_{0}\right)}\right)
$$

Lemma 14. $\mathcal{U}^{\left(i_{0}\right)}$ is a smooth closed hypersurface, namely, for each point $x \in \mathcal{U}^{\left(i_{0}\right)}$ there is a neighborhood of $x$ in which $\mathcal{U}^{\left(i_{1}\right)}$ is defined by the equation $q=\varepsilon_{6}$ and the gradient $\operatorname{grad}_{x}\left(q-\varepsilon_{6}\right)$ does not vanish.

Proof. First we prove that $\operatorname{cl}\left(\mathcal{U}^{\left(i_{0}\right)}\right)=\mathcal{U}^{\left(i_{0}\right)}$. Let a point $x \in \operatorname{cl}\left(\mathcal{U}^{\left(i_{0}\right)}\right)$. Then

$q(x)=\varepsilon_{6}, \quad \varepsilon_{5}^{2} \geq\left(f(x)-\varepsilon_{3}\right)^{2}, \quad u_{i_{0}}^{2}(x) \geq \varepsilon_{4}, \quad p_{1}(x) \geq \varepsilon_{4}, \ldots, p_{s}(x) \geq \varepsilon_{4}$.

None among the latter inequalities could convert into an equality because $q(x)=\varepsilon_{6} \neq 0$, hence $x \in \mathcal{U}^{\left(i_{0}\right)}$.

Since in the open semialgebraic set $\left\{u_{i_{0}}^{2}>0\right\}$ all the square roots (3) are positive, all algebraic functions $p_{1}, \ldots, p_{s}$ occurring in $\mathcal{U}_{0}^{\left(i_{0}\right)}$ are smooth, hence $q$ is smooth as well (see the proof of Lemma 12). Because of Lemma $4, \varepsilon_{6}$ is not a critical value of $q$ in the set $\left\{u_{i, j}^{2}>0\right\}$. The lemma is proved.

Finally, we prove the following lemma.

Lemma 15. The number of local maxima of $L$ on $\mathcal{U}^{\left(i_{0}\right)}$ does not exceed $(n k d)^{o(n)}$. 
Proof. Because of Lemma 14, the number of local maxima of $L$ on $\mathcal{U}^{\left(i_{0}\right)}$ does not exceed the number of connected components of the semialgebraic set

$$
M=\left\{0=q-\varepsilon_{6}=\gamma_{i} \frac{\partial q}{\partial X_{j}}-\gamma_{j} \frac{\partial q}{\partial X_{i}}, 1 \leq i<j \leq n\right\} \subset \mathbf{R}_{6}^{n}
$$

(by the Lagrange multiplier theorem, see, e.g., Chapter 4 in [22] and taking into account the transfer principle).

Replace each occurrence of the square root

$$
\sqrt{u_{i_{0}}^{2}+u_{\pi_{i_{0}(2)}}^{2}+\cdots+u_{\pi_{i_{0}}(m)}^{2}}
$$

$1 \leq m \leq n-i$, in $q$ by a new variable $Z_{m}$. Denote the resulting polynomial by $Q \in \mathbf{R}_{5}\left[X_{1}, \ldots, X_{n}, Z_{1}, \ldots, Z_{n-i}\right]$ (see Section 1 ).

Introduce the semialgebraic set

$$
\begin{aligned}
\mathcal{M}=\left\{0=Q-\varepsilon_{6}=\gamma_{i} \frac{\partial Q}{\partial X_{j}}-\gamma_{j} \frac{\partial Q}{\partial X_{i}}, 1 \leq i<j \leq n,\right. \\
\left.Z_{m}>0, Z_{m}^{2}=u_{i_{0}}^{2}+u_{\pi_{i_{0}(2)}}^{2}+\cdots+u_{\pi_{0}(m)}^{2}, 1 \leq m \leq n-i\right\} \subset \mathbf{R}_{6}^{2 n-i} .
\end{aligned}
$$

Consider the linear projection

$$
\rho: \mathbf{R}_{6}^{2 n-i} \rightarrow \mathbf{R}_{6}^{n}, \quad \rho\left(X_{1}, \ldots, X_{n}, Z_{1}, \ldots, Z_{n-i}\right)=\left(X_{1}, \ldots, X_{n}\right) .
$$

Then $\rho(\mathcal{M})=M$. Hence the number of connected components of $M$ is less than or equal to the number of connected components of $\mathcal{M}$.

Observe that the degrees of polynomials occurring in $\mathcal{M}$ can be bounded from above by $(k n d)^{O(1)}$ due to Lemma 1 and the definition. Therefore, the number of connected components of $\mathcal{M}$ does not exceed $(k n d){ }^{O(n)}$, by [18].

This completes the proof of the lemma.

Lemma 15 together with the corollary to Lemma 13 imply Lemma 10 and thereby Theorems 2 and 1 .

\section{Lower Bounds for Concrete Polyhedra}

In this section we give an application of the lower bound from Theorem 1 to a concrete class of polyhedra. We follow the construction of cyclic polyhedra (see [16]) used in the analysis of the simplex method.

Take any $m>\Omega\left(n^{2}\right)$ points in $\mathbf{R}^{n}$ of the form $\left(t_{j}, t_{j}^{2}, \ldots, t_{j}^{n}\right)$ for pairwise distinct $t_{j}$, $1 \leq j \leq m$. Consider the convex hull of these points and denote by $P_{n, m} \subset \mathbf{R}^{n}$ its dual polyhedron [16]. Then $P_{n, m}$ has $m$ faces of the highest dimension $n-1$ and the number of faces of all dimensions is

$$
N>\left(\begin{array}{c}
m-\lfloor n / 2\rfloor \\
\lfloor n / 2\rfloor
\end{array}\right)>m^{\Omega(n)}
$$


(see [16]). Therefore, Theorem 1 implies that the complexity of testing membership to $P_{n, m}$ is bounded by $\Omega(\log N)>\Omega(n \log m)$.

We would like to mention that Section 4 of [9] provides a weaker bound $\Omega(\log m)$ even for algebraic computation trees.

\section{Complexity Lower Bound for Algebraic Computation Trees}

Now we show how to extend the obtained complexity lower bound from decision to computation trees and, thereby, prove Theorem $1^{\prime}$.

Let an algebraic computation tree $T$ of depth $k$ test membership to a polyhedron $P$. Fix some branch in $T$ with the output "yes," and denote by $f_{1}, \ldots f_{k} \in \mathbf{R}\left[X_{1}, \ldots X_{n}\right]$ the testing polynomials along this branch. Then there is a sequence of polynomials $h_{1}, \ldots, h_{k}$ such that $h_{i} \in \mathbf{R}\left[X_{1}, \ldots, X_{n}, Z_{1}, \ldots, Z_{i-1}\right]$ with $\operatorname{deg}\left(h_{i}\right) \leq 2$ and $f_{i}=$ $h_{i}\left(X_{1}, \ldots X_{n}, f_{1}, \ldots, f_{i-1}\right)$ for $1 \leq i \leq k$. (In fact the polynomials $h_{i}$ correspond to the elementary arithmetic operations and have a simple structure, but we use only that $\operatorname{deg}\left(h_{i}\right) \leq 2$ ). For this branch a semialgebraic set

$$
W=\left\{f_{1} \sigma_{1} 0, \ldots, f_{k} \sigma_{k} 0\right\} \subset P
$$

of the accepted points is assigned, where $\sigma_{1}, \ldots, \sigma_{k} \in\{=,>\}$ (see the Introduction).

Consider a semialgebraic set

$$
\mathcal{W}=\left\{Z_{i}=h_{i}\left(X_{1}, \ldots, X_{n}, Z_{1}, \ldots, Z_{i-1}\right), 1 \leq i \leq k ; Z_{1} \sigma_{1} 0, \ldots, Z_{k} \sigma_{k} 0\right\} \subset \mathbf{R}^{n+k}
$$

which can be treated as the "graph" of the branch. Denote by $\pi: \mathbf{R}^{n+k} \rightarrow \mathbf{R}^{n}$ the natural projection and by $\rho: \mathbf{R}^{n} \rightarrow \mathbf{R}^{n+k}$ the (graph) mapping $\rho(x)=\left(x, f_{1}(x), \ldots, f_{k}(x)\right)$. Then $\pi(\mathcal{W})=W$ and $\rho(W)=\mathcal{W}$, therefore the semialgebraic sets $W$ and $\mathcal{W}$ are polynomially isomorphic. By the same token, the polyhedron $P$ is isomorphic to its $\rho$-image $\mathcal{P}=\rho(P)$. Actually, $\mathcal{P}$ satisfies the following definition.

Definition. A subset $\mathcal{P}_{1} \subset \mathbf{R}^{m}$ is called a distorted (closed) polyhedron if there exist polynomials $g_{1}, \ldots, g_{s} \in \mathbf{R}\left[X_{1}, \ldots, X_{m}\right]$ such that:

(1) Zero is a noncritical value of $g_{i}$ for every $1 \leq i \leq s$.

(2) The smooth hypersurfaces $\left\{g_{i}=0\right\}$ intersect transversally, i.e., at any point $x \in \mathbf{R}^{m}$ the gradient vectors $\operatorname{grad}\left(g_{i_{j}}\right)(x)$ are linearly independent for all $i_{j}$ such that $g_{i_{j}}(x)=0$.

Finally,

$$
\mathcal{P}_{1}=\left\{g_{1} \geq 0, \ldots, g_{s_{1}} \geq 0, g_{s_{1}+1}=\cdots=g_{s}=0\right\} .
$$

A (distorted) face of $\mathcal{P}_{1}$ is a connected component of a (nonempty) semialgebraic set of the form

$$
\left\{g_{i}=0, i \in I ; g_{j}>0, j \notin I ; g_{s_{1}+1}=\cdots=g_{s}=0\right\} \subset \mathcal{P}_{1}
$$

for a subset of indices $I \subset\left\{1, \ldots, s_{1}\right\}$. 
We say that a semialgebraic set

$$
\mathcal{W}_{1}=\left\{f_{1}^{\prime}=\cdots=f_{t_{1}}^{\prime}=0, f_{t_{1}+1}^{\prime}>0, \ldots, f_{t}^{\prime}>0\right\} \subset \mathcal{P}_{1}
$$

with $\operatorname{deg}\left(f_{j}^{\prime}\right) \leq d$ touches a (distorted) face $p_{1}$ of $\mathcal{P}_{1}$ if $\operatorname{dim}\left(p_{1} \cap \mathcal{W}_{1}\right)=\operatorname{dim}\left(p_{1}\right)$. Similar to the proof of the Theorem 2 the following bound could be proved.

Theorem 2'. The number of faces of $\mathcal{P}_{1}$ touched by $\mathcal{W}_{1}$ does not exceed $(\mathrm{dtm})^{O(m)}$.

Observe that $\mathcal{P} \subset \mathbf{R}^{n+k}$ is a distorted polyhedron. We apply Theorem $2^{\prime}$ to $\mathcal{P}$ and the set $\mathcal{W}$, taking into account that a face $p$ of $P$ is touched by $W$ if and only if the (distorted) face $\rho(p)$ of $\mathcal{P}=\rho(P)$ is touched by $\rho(W)=\mathcal{W}$. Therefore, the number of faces of $P$ touched by $W$ does not exceed $(4 k(n+k))^{O(n+k)} \leq(k n)^{O(n+k)}$. Finally, as in the Introduction, we get the upper bound $N<3^{k}(k n)^{O(n+k)} \leq(k n)^{O(n+k)}$ on the number $N$ of the faces of $P$ which implies the lower bound $k \geq \Omega(\log N / \log \log N)$ provided that $N>n^{c_{1} n}$ for a suitable $c_{1}$, and, thus, Theorem $1^{\prime}$. Note that if the restriction $N>n^{c_{1} n}$ is not fulfilled, the bound $\log N / \log \log N$ grows slower than $O(n)$.

An interesting open problem is to eliminate the denominator $\log \log N$ in the lower bound.

\section{Acknowledgments}

We thank Dima Burago, Friedhelm Meyer auf der Heide, Kolia Ivanov, and Andy Yao for a number of useful discussions and Anders Björner for a help with the example from Section 6.

\section{References}

1. M. Ben-Or, Lower bounds for algebraic computation trees, Proceedings of ACM Symposium on Theory of Computing, 1983, pp. 80-86.

2. A. Björner, Subspace arrangements, Proceedings of 1 st European Congress of Mathematics, Paris, 1992, A. Joseph et al., eds., Progress in Mathematics, Vol. 119, Birkhäuser, Basel, 1994, pp. 321-370.

3. A. Björner and L. Lovasz, Linear decision trees, subspace arrangements and Möbius functions, Preprint, 1992.

4. A. Björner, L. Lovasz, and A. Yao, Linear decision trees: Volume estimates and topological bounds, Proceedings of ACM Symposium on Theory of Computing, 1992, pp. 170-177.

5. P. Buergisser, M. Karpinski, and T. Lickteig, On randomized algebraic test complexity, Technical Report TR-92-070, International Computer Science Institute, Berkeley, CA, 1992.

6. M. Goresky and R. MacPherson, Stratified Morse Theory, Springer-Verlag, Berlin, 1988.

7. D. Grigoriev, Complexity of deciding Tarski algebra, J. Symbolic Comput., 5 (1988), 65-108.

8. D. Grigoriev, M. Karpinski, and M. Singer, Computational complexity of sparse real algebraic function interpolation, Proceedings MEGA '92, Progress in Mathematics, Vol. 109, Birkhäuser, Basel, 1993. pp. 91-104.

9. D. Grigoriev, M. Karpinski, and N. Vorobjov, Lower bounds on testing membership to a polyhedron by algebraic decision trees, Proceedings of ACM Symposium on Theory of Computing, 1994, pp. 635-644.

10. D. Grigoriev and N. Vorobjov, Solving systems of polynomial inequalities in subexponential time, J. Symbolic Comput., 5 (1988), 37-64. 
11. D. Grigoriev and N. Vorobjov, Counting connected components of a semialgebraic set in subexponential time, Comput. Complexity, 2 (1992), 133-186.

12. D. Grigoriev and N. Vorobjov, Complexity lower bounds for computation trees with elementary transcendental function gates, Proceedings of IEEE Symposium on Foundations of Computer Science, 1994, pp. 548-552. Also in Theoret. Comput. Sci., 157 (1996), 185-214.

13. M. Hirsch, Differential Topology, Springer-Verlag, New York, 1976.

14. S. Lang, Algebra, Addison-Wesley, Reading, MA, 1965.

15. R. Loos, Generalized polynomial remainder sequences, in Symbolic and Algebraic Computation, B. Buchberger et al., eds., Springer-Verlag, New York, 1982, pp. 115-136.

16. P. McMullen and G. Shephard, Convex Polythopes and the Upper Bound Conjecture, Cambridge University Press, Cambridge, 1971.

17. F. Meyer auf der Heide, Fast algorithms for $n$-dimensional restrictions of hard problems, Proceedings of ACM Symposium on Theory of Computing, 1985, pp. 413-420.

18. J. Milnor, On the Betti numbers of real varieties, Proc. Amer. Math. Soc. 15 (1964), 275-280.

19. J. L. Montaña, J. E. Morais, and L. M. Pardo, Lower bounds for arithmetic networks, 2: sum of Betti numbers, Appl. Algebra Engrg. Comm. Comput. 7 (1996), 41-51.

20. M.-F. Roy and N. Vorobjov, Finding irreducible components of some real transcendental varieties, Comput. Complexity 4 (1994), 107-132.

21. A. Tarski, A Decision Method for Elementary Algebra and Geometry, University of California Press, Berkeley, CA, 1951.

22. J. A. Thorpe, Elementary Topics in Differential Geometry, Springer-Verlag, Berlin, 1977.

23. A. Yao, Algebraic decision trees and Euler characteristics, Proceedings of IEEE Symposium on Foundations of Computer Science, 1992, pp. 268-277.

24. A. Yao, Decision tree complexity and Betti numbers, Proceedings of ACM Symposium on Theory of Computing, 1994, pp. 615-624.

25. A. Yao and R. Rivest, On the polyhedral decision problem, SIAM J. Comput. 9 (1980), 343-347.

Received December 27, 1994, and in revised form April 15, 1996. 